\title{
Prognostics and Health Management for Maintenance Practitioners - Review, Implementation and Tools Evaluation
}

\author{
Vepa Atamuradov ${ }^{1}$, Kamal Medjaher ${ }^{1}$, Pierre Dersin ${ }^{2}$, Benjamin Lamoureux ${ }^{2}$ and Noureddine Zerhouni ${ }^{3}$. \\ ${ }^{1}$ Production Engineering Laboratory (LGP), INP-ENIT 47 Av. d'Azereix, 65000 Tarbes, France \\ vepa.atamuradov@enit.fr \\ kamal.medjaher@enit.fr \\ ${ }^{2}$ ALSTOM Transport, 93400 Saint-Ouen, France \\ pierre.dersin@transport.alstom.com \\ benjamin.lamoureux@transport.alstom.com \\ ${ }^{3}$ FEMTO-ST Institute, UMR CNRS 6174 - UFC/ENSMM 15B av. des Montboucons, 25000 Besancon, France \\ zerhouni@ens2m.fr
}

\begin{abstract}
In literature, prognostics and health management (PHM) systems have been studied by many researchers from many different engineering fields to increase system reliability, availability, safety and to reduce the maintenance cost of engineering assets. Many works conducted in PHM research concentrate on designing robust and accurate models to assess the health state of components for particular applications to support decision making. Models which involve mathematical interpretations, assumptions and approximations make PHM hard to understand and implement in real world applications, especially by maintenance practitioners in industry. Prior knowledge to implement PHM in complex systems is crucial to building highly reliable systems. To fill this gap and motivate industry practitioners, this paper attempts to provide a comprehensive review on PHM domain and discusses important issues on uncertainty quantification, implementation aspects next to prognostics feature and tool evaluation. In this paper, PHM implementation steps consists of; (1) critical component analysis, (2) appropriate sensor selection for condition monitoring (CM), (3) prognostics feature evaluation under data analysis and (4) prognostics methodology and tool evaluation matrices derived from PHM literature. Besides PHM implementation aspects, this paper also reviews previous and on-going research in high-speed train bogies to highlight problems faced in train industry and emphasize the significance of PHM for further investigations.
\end{abstract}

\section{INTRODUCTION}

Reliability, availability, safety and maintenance cost effectiveness have been an important concern in many

\footnotetext{
Vepa Atamuradov et al. This is an open-access article distributed under the terms of the Creative Commons Attribution 3.0 United States License, which permits unrestricted use, distribution, and reproduction in any medium, provided the original author and source are credited.
}

industries. Company profit and competitiveness depend on designing and producing highly reliable systems and/or good quality products. However, designing such sophisticated systems brings about many difficulties and concerns such as component maintenance cost, along with its merits. For industry, an important issue is how to reduce maintenance cost and manage business risks while increasing asset reliability, availability, and safety. Hence there is a need to design and develop sophisticated health assessment technologies, such as PHM, which are easily implementable by maintenance technicians or engineers to improve component reliability and safety.

The Prognostics and Health Management (PHM) discipline provides for viewing overall health state of machines or complex systems and assists in making correct decisions on machine maintenance. The main duties of PHM technology are to detect incipient component or system fault, perform failure diagnostics, failure prognostics, and health management. There are three main issues to be considered when building a robust PHM: an estimation of current health state, prediction of a future state along with time to fail, and determination of a failure's impact on the performance of a system. For practitioners, to select and implement PHM technology is based on their ability and knowledge about PHM approaches, tools, etc. Models which involve mathematical interpretations, assumptions and approximations make PHM hard to understand and apply. Prior knowledge to implement PHM in complex systems is crucial to building highly reliable systems.

The goal of this current review paper is to serve as a handbook for practitioners in industry to motivate and assist them through PHM implementation and help them to accomplish their duty more easily. In this paper, a four-step maintenance assessment approach is designed which consists of (1) critical component analysis, (2) right sensor selection for CM, (3) prognostics feature evaluation and (4) prognostics methodology and tool evaluation matrices derived from PHM approaches presented in the literature. 
Besides PHM approaches literature review and proposed an approach, this paper also reviews previous and on-going research in high-speed train bogies to highlight the problems faced in the railway industry and to emphasize the significance of PHM for further investigations. There are several review papers in literature conducted in fault diagnostics and prognostics techniques. Condition Based Maintenance (CBM), which is one of the frequently studied and applied maintenance techniques in many engineering applications, was reviewed in (Jardine, Lin, \& Banjevic, 2006). Authors studied implementation issues of CBM for mechanical systems and reviewed diagnostics and prognostics developments, data preprocessing algorithms and data fusion techniques in the literature. Rotating machinery prognostics articles were reviewed and synthesized in (Heng, Zhang, Tan, \& Mathew, 2009). Failure prediction methodologies for rotating machinery were classified as reliability models, condition based and integrated models with their merits and drawbacks. Sikorska and M. Hodkiewiczb (2011) reviewed RUL estimation and prognostics modeling approaches under knowledge-based, life expectancy, artificial neural networks (ANN) and physics models. Authors presented prognostics models with their limitations and merits and discussed an appropriate model selection for business cases. J. Lee et al. (2014), presented comprehensive review, conducting in PHM of rotatory machinery and introduced a $5 \mathrm{~S}$ systematic methodology for PHM design which was evaluated in different industrial case studies. Kandukuri et al. (2016) reviewed diagnostics and prognostics methodologies under reliability centered maintenance (RCM) and CBM for two critical components; planetary gearboxes and low-speed bearings of wind turbines. Authors analyzed different CM data for bearing and gearbox diagnostics and concluded that vibration signal was better in gearbox fault detection and acoustic emission for bearing fault detection. Furthermore, CBM based on RCM as an ideal maintenance strategy was suggested for farm-level wind turbine health assessment. Guillén et al. (2013) studied PHM integration framework main topics regarding monitoring and diagnostics techniques by synthesizing PHM review papers in different application areas. Then, they discussed PHM functionality, maintenance types, prognostics approaches and proposed the integration of PHM with e-maintenance for proactive decision making. State of the art of PHM for nuclear power plants (NNP) was presented in (Coble et al. 2015) where they reviewed prognostics and maintenance techniques for active and passive NNP components. An Kim, and Choi (2015) reviewed a data-driven and physics-based prognostics algorithms in terms of model definition, parameter estimation, robustness in noise and bias handling in $\mathrm{CM}$ data, to provide practical prognostics options for beginners. Kan, Tan, and Mathew (2015) reviewed datadriven prognostics techniques for non-linear and nonstationary machine processes. The authors studied prognostics techniques that can cope with non-linearity and non-stationarity and concluded with further improvements in prognostics. In (Rezvanizaniani, Liu, Chen, \& Lee, 2014) authors reviewed a techniques for the battery PHM approaches to provide cost effective solutions for battery health assessment. These reviewed papers can be used to understand the general concepts of system CM. In Table 1, we have summarized important issues and remarks pointed out by the review papers synthesized from the literature.

\begin{tabular}{|c|c|c|c|}
\hline Authors & Domain & Approach Classification & Issues \& Remarks \\
\hline Jardine et al. (2006) & Mechanical & $\begin{array}{l}\text { - Physics-based } \\
\text { - Data-driven } \\
\text { - }\end{array}$ & $\begin{array}{l}\text { - Lack of communication between scientists } \\
\text { and industry practitioners } \\
\text { - Lack of data collection and efficient } \\
\text { approach validation } \\
\text { - Implementation difficulty due to rapid } \\
\text { change of technology design and } \\
\text { management executives }\end{array}$ \\
\hline Heng et al. (2009) & Mechanical & $\begin{array}{l}\text { - Physics-based } \\
\text { - Data-driven }\end{array}$ & $\begin{array}{l}\text { - Integration of CM with event data (ED) } \\
\text { - Proper usage of incomplete data } \\
\text { - Maintenance action effects } \\
\text { - Machine operating conditions } \\
\text { - Analysis of nonlinearity between } \\
\text { condition monitoring data and component } \\
\text { health state } \\
\text { - Consideration of different failure } \\
\text { interactions } \\
\text { - Practical requirements and assumptions } \\
\text { - Developing uniform health measurement } \\
\text { framework }\end{array}$ \\
\hline Sikorska et al. (2011) & Mechanical & $\begin{array}{l}\text { - Knowledge-based } \\
\text { - Life expectancy } \\
\text { - Physics-based }\end{array}$ & $\begin{array}{l}\text { - Prediction requirements; the goal of RUL } \\
\text { prediction } \\
\text { - Model-process compatibility; } \\
\text { compatibility of the model with the real } \\
\text { world. }\end{array}$ \\
\hline
\end{tabular}




\begin{tabular}{|c|c|c|c|}
\hline & & & $\begin{array}{l}\text { - Resource requirements; availability of } \\
\text { resources to undertake the modeling (data, } \\
\text { skills/experience) } \\
\text { - Approach readiness; proof of model } \\
\text { reliability. }\end{array}$ \\
\hline Lee et al. (2014) & Mechanical & $\begin{array}{l}\text { - Physics-based } \\
\text { - Data-driven } \\
\text { - Hybrid }\end{array}$ & $\begin{array}{l}\text { - Stated importance of maintenance strategy } \\
\text { transformation from fail-and-fix to the } \\
\text { predict-and-prevent methodology. } \\
\text { - PHM was treated as evolved form of } \\
\text { CBM. } \\
\text { - Future trends of PHM were concluded as } \\
\text { self-maintenance, resilient systems, and } \\
\text { engineering immune system. }\end{array}$ \\
\hline Kandukuri et al. (2016) & Renewable energy & - Data-driven & $\begin{array}{l}\text { - Suggested CBM based RCM as an ideal } \\
\text { maintenance strategy for wind turbine } \\
\text { - Concluded that vibration signal was better } \\
\text { in gearbox fault detection and acoustic } \\
\text { emission for bearing fault detection } \\
\end{array}$ \\
\hline Guillén et al. (2013) & Mechanical & $\begin{array}{l}\text { - Physics-based } \\
\text { - Data-driven } \\
\text { - Hybrid }\end{array}$ & $\begin{array}{l}\text { - Integration of PHM with } \\
\text { e-maintenance for proactive decision } \\
\text { making was emphasized. }\end{array}$ \\
\hline Coble et al. (2015) & Nuclear plants & $\begin{array}{l}\text { - Physics-based } \\
\text { - Data-driven }\end{array}$ & $\begin{array}{l}\text { Authors stated followings for detecting and } \\
\text { managing degradation of reactor } \\
\text { components; } \\
\text { - Developing non-destructive measurement } \\
\text { methods and analysis for anomaly } \\
\text { detection, } \\
\text { - Developing monitoring algorithms for } \\
\text { component degradation } \\
\text { - Developing prognostics tools for RUL } \\
\text { estimation. }\end{array}$ \\
\hline An et al. (2015) & Mechanical & $\begin{array}{l}\text { - Physics-based } \\
\text { - Data-driven }\end{array}$ & $\begin{array}{l}\text { - Model definition } \\
\text { - Parameter estimation } \\
\text { - Robustness in noise and bias handling in } \\
\text { CM data was studied to provide practical } \\
\text { solutions for machine prognostics }\end{array}$ \\
\hline Kan et al. (2015) & Mechanical & $\begin{array}{l}\text { - Model-based } \\
\text { - Data-driven } \\
\text { - Combination models }\end{array}$ & $\begin{array}{l}\text { - Lack of run-to-failure historical data } \\
\text { - Improving prognostics techniques' } \\
\text { feasibility with minimum uncertainty } \\
\text { - Good model validation establishment and } \\
\text { simplification of techniques for real-time } \\
\text { prognostics } \\
\text { - Developing global prognostics technique } \\
\text { ranking system to compare their } \\
\text { performances for all kind of machinery }\end{array}$ \\
\hline $\begin{array}{l}\text { Rezvanizaniani et al. } \\
(2014)\end{array}$ & Electrical & $\begin{array}{l}\text { - Model-based } \\
\text { - Data-driven } \\
\text { - Fusion models }\end{array}$ & $\begin{array}{l}\text { - Compared different approach drawbacks } \\
\text { - The main challenges in battery health } \\
\text { management were summarized as } \\
\text { uncertainty in mobility, durability and } \\
\text { safety }\end{array}$ \\
\hline
\end{tabular}

Table 1. Main issues \& remarks studied by review papers in literature.

The paper is organized as follows: In Section 2, PHM main tasks are explained to introduce general ideology. Section 3 presents PHM implementation challenges and/or issues System-level based PHM is presented in Section 4. Section 5 presents uncertainty quantification. Section 6 reviews component-level PHM approaches and prognostics tools. Bogie component diagnostics and prognostics techniques are reviewed in Section 7. Section 8 contains PHM implementation steps for industry and Section 9 concludes the paper.

\section{PHM MAIN TASKS}

In this section, PHM implementation steps are discussed and explained in detail to instruct practitioners and make them familiar with PHM infrastructure. Steps involve data acquisition, data preprocessing, detection, diagnostics and prognostics, decision making and finally human-machine interface. PHM steps are depicted in Fig. 1. Each step will be explained in the following subsections. 


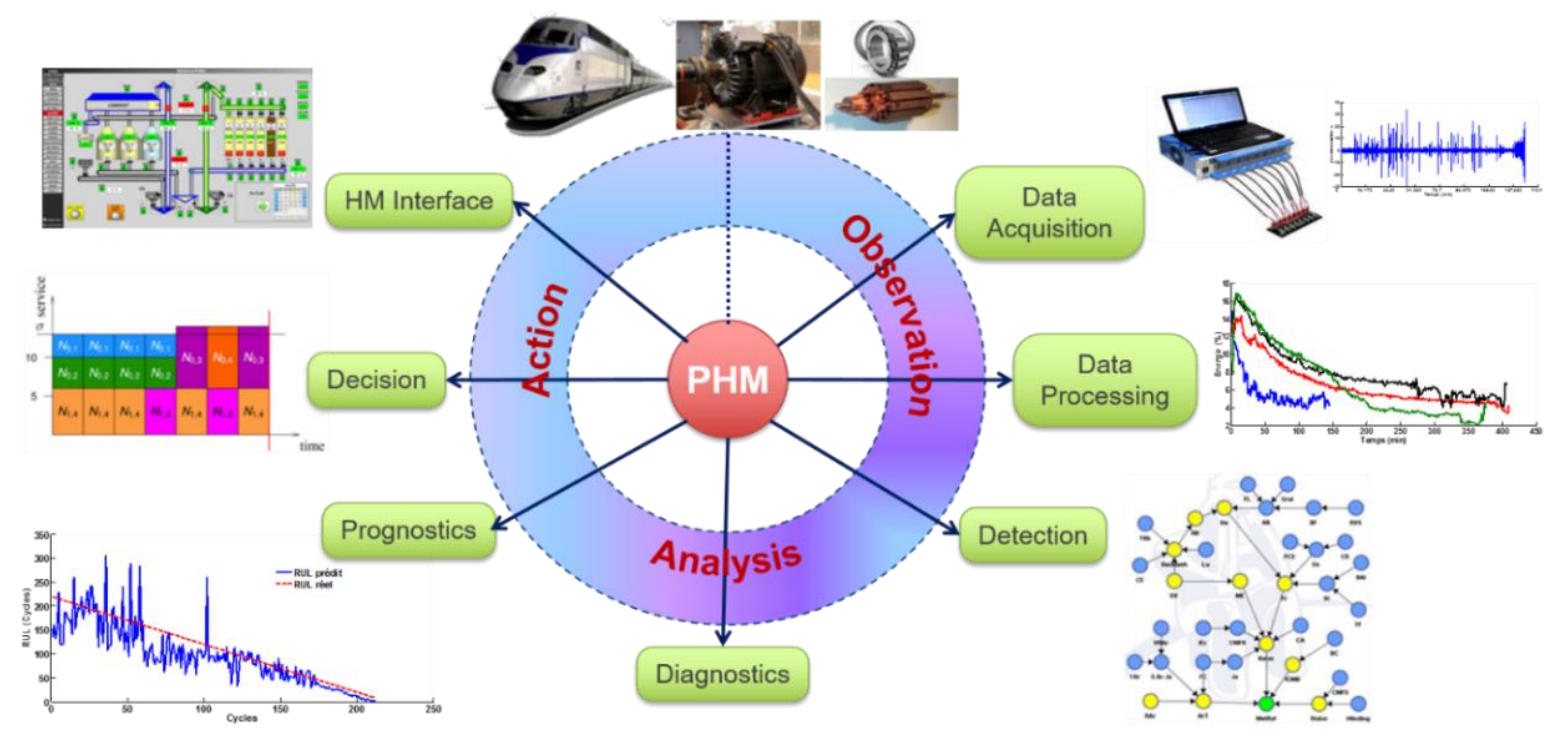

Fig. 1. PHM steps.

\subsection{Data Acquisition}

Data acquisition is an initial and essential step of PHM which is known as a process of data collection and storage from physical component/system under investigation for further diagnostics and prognostics purposes. Collected data could be either sensory data or event data (ED). The ED include the information of maintenance actions (e.g. oil change, repairs etc.) taken on the events (e.g. failure, breakdown, installation etc.) that happened to the physical component. CM or sensory data are measurements tracked via installed sensors from asset under investigation, such as; acoustic emission data, vibration data, temperature, pressure, humidity, resistance, voltage, etc. Where ED includes events performed by maintenance technician, such as corrective maintenance, asset repairs, installation, breakdown, cleaning and oiling on the component/system. Data acquisition process is depicted in Fig. 2 for a railway point machine example. ISO definition for data acquisition can be found in (ISO 13374-1:2003, n.d.).

\subsection{Data Preprocessing}

Data preprocessing involves data cleaning and data analysis steps. Cleaning errors/noise from raw data increases the chance of getting error-free data for further investigations. Data analysis, which is the second step of data preprocessing, involves feature extraction, feature evaluation, and selection processes. Cleaned sensory time series should undergo a feature extraction process to extract only the important and useful features that reflect system health state being monitored. Extracted features should indicate the failure progression of the system. The feature extraction techniques are categorized as time-domain based, frequency-based and time-frequency based techniques in the literature (Jardine et al., 2006). The timedomain based feature extraction techniques (e.g. root mean square, kurtosis etc.) are used to analyze the global characteristics of data and to extract the features in time domain. The frequency-domain based feature extraction techniques (e.g. Fourier transform, envelop analysis etc.) transform the data into frequency domain and are used to detect and identify a faults which are not possible by timedomain based techniques. The time-frequency domain based techniques (e.g. Fourier transform, envelop analysis etc. Hilbert-Huang transform, Wigner-Ville distribution etc.) analyze the data in both time and frequency domains. Feature evaluation and selection process is the second important step of data analysis after extraction. A feature evaluation can be defined as a feature goodness quantification process in feature selection. There are different techniques used to quantify the feature goodness (i.e. degradation trend) such as monotonicity, prognosability and trendability (J. Coble \& Hines, 2009). The best features, which have clear degradation trend, are further selected in a feature selection process after evaluation (Kimotho \& Sextro, 2014). More information on feature extraction techniques can be found in (Sharma \& Parey, 2016; Zhu, Nostrand, Spiegel, \& Morton, 2014) articles.

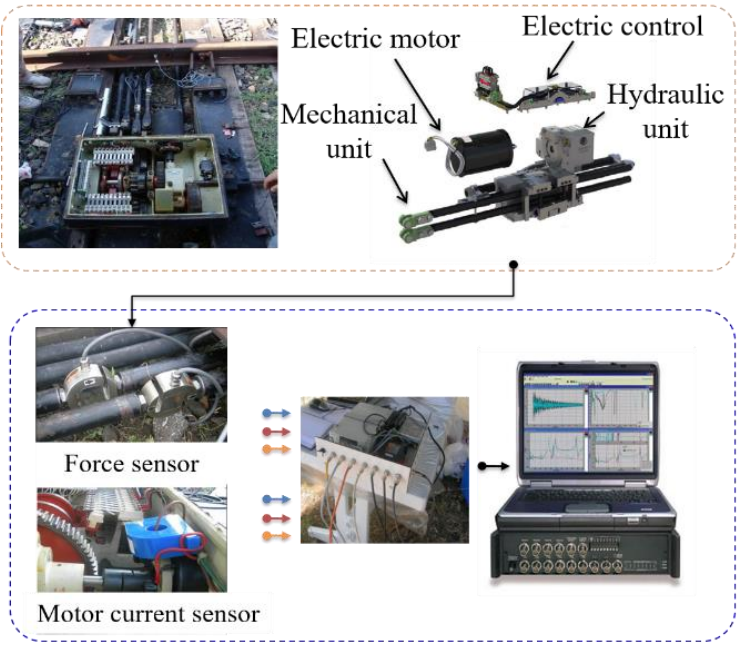

Fig. 2. Data acquisition process for railway point machine. 


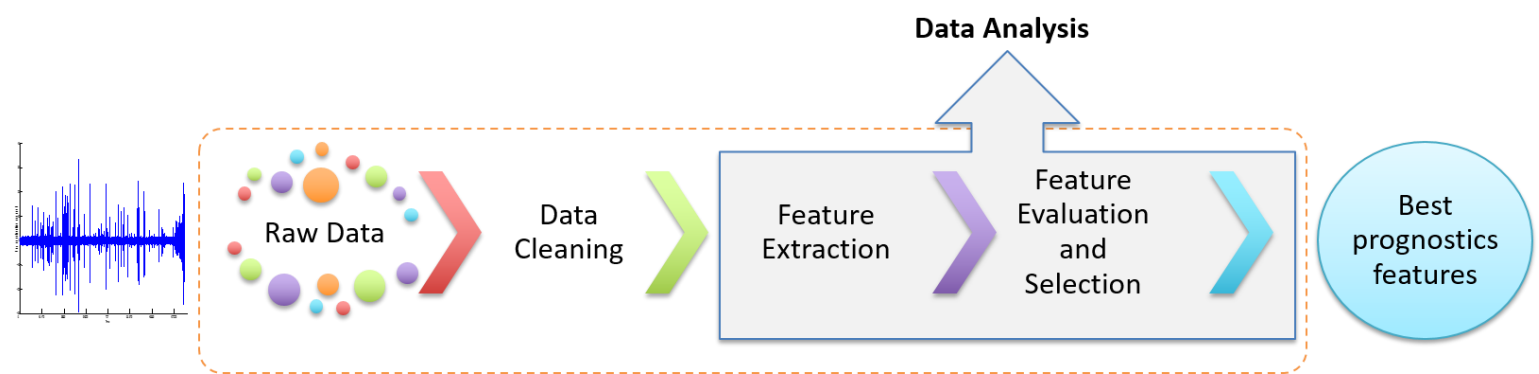

Fig. 3. Data preprocessing procedure.

An accurate prediction of remaining-useful-life (RUL) of assets depends on well evaluated and selected prognostic features. Overall data preprocessing procedure is depicted in Fig. 3. ISO definition for data preprocessing can be found in (ISO 13374-1:2003, n.d.).

\subsection{Detection}

There are many factors that cause system components to degrade over time, losing their initial performance, and which therefore need to be considered in detection modeling. Health state detection is the process of detecting and recognizing incipient failures and/or anomalies from CM data. A fault detection is typically based on the quantification of the inconsistencies between the actual and the expected behavior of the system in nominal conditions. Fig. 4 illustrates failure propagation of component based on CM data.

In Fig. 4, a component CM indicator increases with time as the component degrades. As illustrated in the figure, the evolution of the health state of the component can be divided into 3 phases; Phase-1 $\left(\mathrm{T}_{0}<\mathrm{T}_{1}\right)$ where the component is in a healthy state, Phase- $2\left(\mathrm{~T}_{1}<\mathrm{T}_{2}\right)$ where the component is in a faulty state, Phase- $3\left(\mathrm{~T}_{2}<\right)$ where the component is in the completely failed state. Predefining thresholds $\left(T_{1}\right.$ and $\left.T_{2}\right)$ is very challenging, which needs serious experience for practitioners. Historical CM data can be also used to compare and set new thresholds for the same type of components. Time-to-failure estimation is performed in Phase- 2 after the detection process where maintenance activities are planned based on estimated time-to-failure. Therefore, early detection of component failure is important. Fig. 5 illustrates features' health state

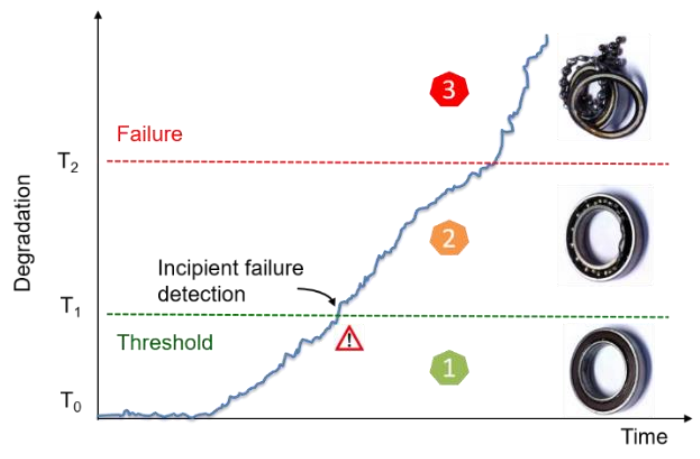

Fig. 4. Component failure propagation.

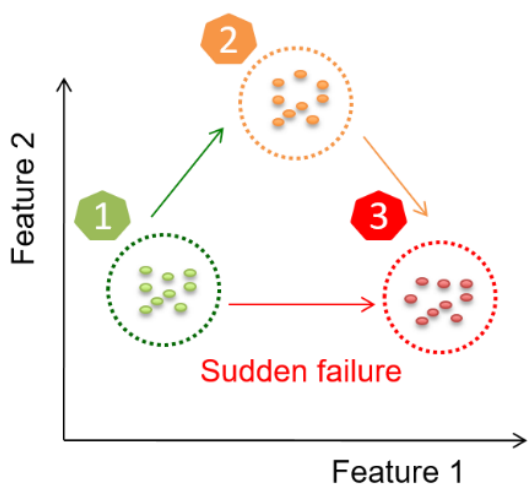

Fig. 5. Feature health state transitions.

transitions for slowly propagating failures (healthy-faultyfailed) and for sudden failures (healthy-failed). ISO definition for fault detection can be found in (ISO 13374$1: 2003$, n.d.).

\subsection{Diagnostics}

Fault diagnostics is a process of fault detection, isolation (i.e. which component is failed), failure mode identification (i.e. what is the cause of failure or fault) and degradation level assessment (i.e. quantification of the failure severity) in condition monitoring. Diagnostics can be conducted when a machine is either in complete failure state or in faulty state. Fig. 6 illustrates the post-mortem fault diagnostics for failed component. Diagnostics results can be used for reactive as well as proactive decision making (the latter when diagnosing a degraded condition, as opposed to a complete failure). ISO definition for fault diagnostics can be found in (ISO 13372:2012, n.d.).

\subsection{Prognostics}

Prognostics is defined as the process of predicting the time (RUL) at which a component will no longer perform a particular function and it is illustrated in Fig. 7. Prognostics results are used to support proactive decision making. ISO definition for fault prognostics can be found in (ISO 13381$1: 2005$, n.d.).

As illustrated in two examples (Fig. 6 and Fig. 7), prognostics serves as prevention of system from possible failures by predicting future states while diagnostics is 
concerned with fault isolation and classification process. Prognostics and diagnostics difference is depicted in Fig. 8.

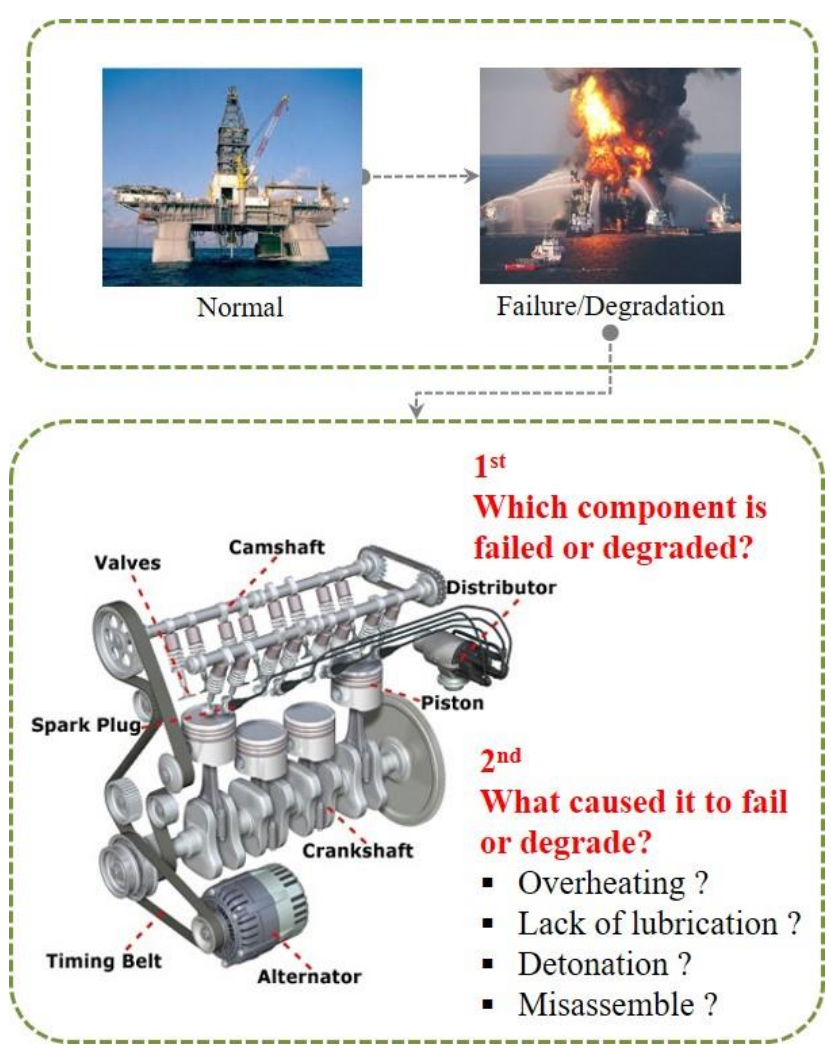

Fig. 6. Diagnostics.
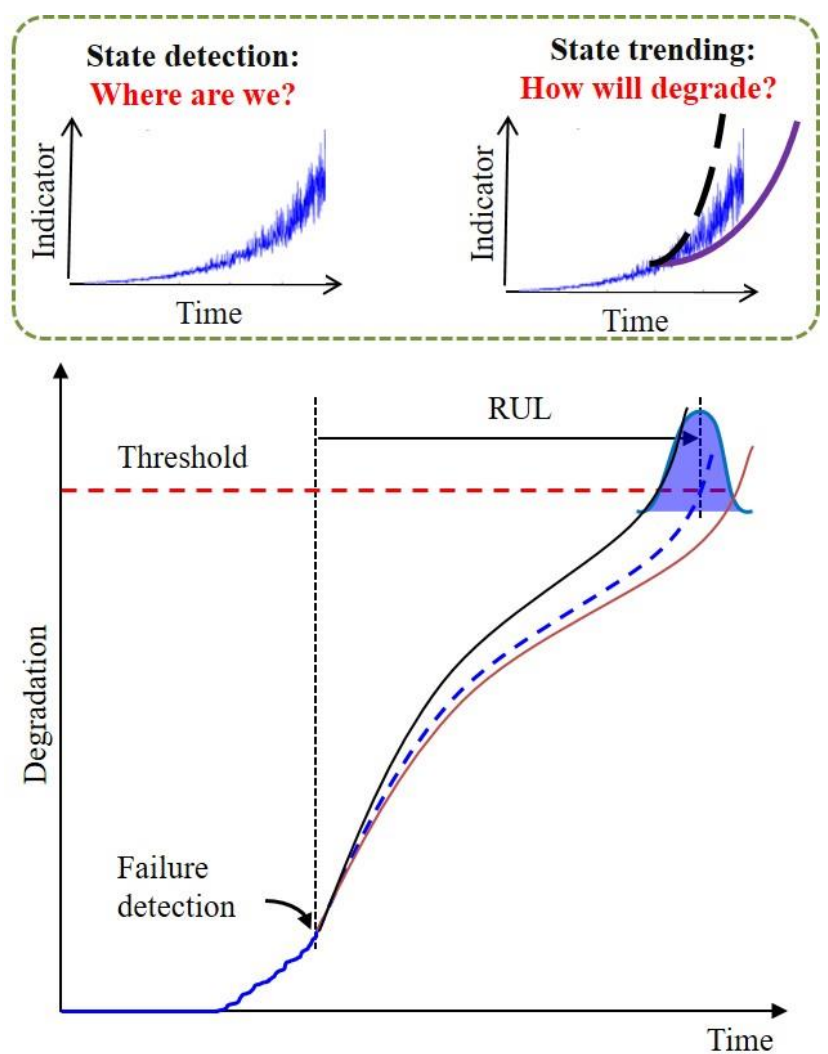

Fig. 7. Prognostics.

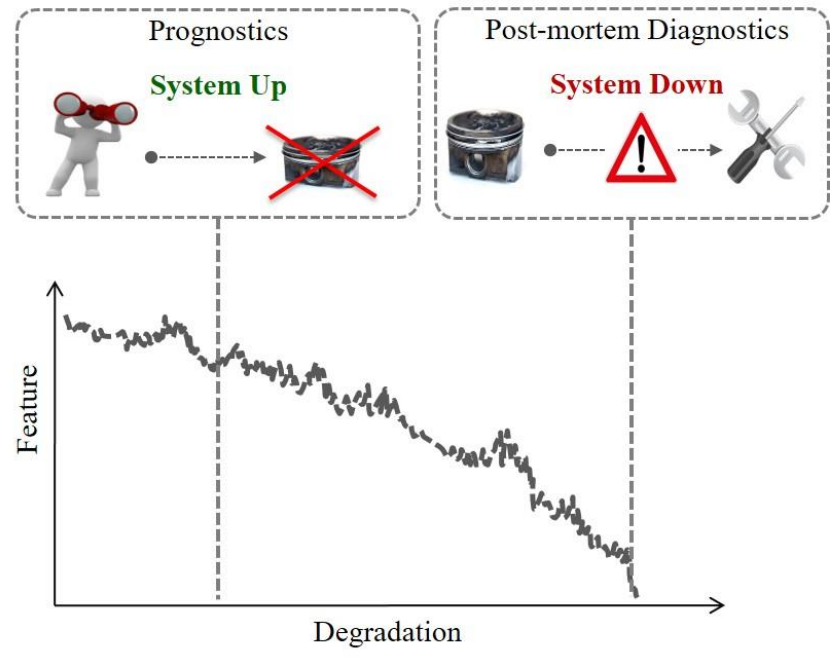

Fig. 8. Prognostics vs. Diagnostics.

\subsection{Decision Making}

Decision making is a process resulting in the selection of logical and/or right maintenance action among several alternatives. Maintenance technician must evaluate the negatives and positives of each action based on the diagnostics or prognostics results. To make effective decisions, the technician also should be able to estimate the outcomes of each alternative as well. ISO definition for decision making can be found in (ISO 13374-1:2003, n.d.)

Outcomes of decisions could be either operational or design based. Decisions made on operational actions could be maintenance interventions, hardware/software reconfigurations and fault tolerant control (FTC). Design based outcomes could be adding and/or replacing sensors observability and redesign and/or components placement. Decision-making process is illustrated in Fig. 9.

\subsection{Human-Machine Interface}

Human-machine interface is Graphical User Interface (GUI) which is used to visualize component health status, to execute tasks, to analyze data and to control the maintenance operations.

\section{PHM CHALLENGES}

This section will summarize general and step based challenges of PHM. PHM and its implementation is a very challenging task that requires several aspects in need for further investigation before applying it reliably in real applications. Hence, it is important to develop sophisticated reliable degradation models for the accurate estimation and prediction of its evolution. Safety-critical systems such as; automotive, train, nuclear, chemical and aerospace industries, need smart predictive maintenance systems with very high reliability due to potential catastrophic failure consequences. Due to this, smart and reliable PHM technology development is urgent to cope with maintenance optimization tasks of critical complex systems efficiently. Thus, developed PHM system should 
take into account asset and system degradation, environmental effects, failure behaviors, failure interactions and related uncertainties (Qiao \& Weiss, 2016). These PHM challenges are illustrated in Fig. 10. Further challenges faced in PHM steps are data acquisition, data preprocessing, detection, diagnostics and prognostics and decision making. Challenges in PHM steps are synthesized in Fig. 11.

\section{SySTEM-LEVEL PHM}

In this section, scientific works conducted toward systemlevel based PHM approaches are reviewed and challenges in implementation steps are discussed.

Generally, most of the research conducted in failure diagnostics and prognostics of assets in literature are based on component-level health assessment. However, complex engineering systems are composed of multiple and interactively functioning individual components that can influence the system performance very seriously when they fail. Hence, development of PHM methodologies for system-level monitoring is also important as well as component-level PHM methodologies (Sankavaram et al., 2016). (Khorasgani, Biswas, \& Sankararaman, 2016) proposed comprehensive two-step analytic system-level prognostics methodology for rectifier systems which consist of estimation and prediction step. In estimation step, performance degradation models of individual components were combined and were adopted into particle filtering $(\mathrm{PF})$ based estimation. Whereas in the prediction step, first order reliability method (FORM) based systemlevel prediction model was built to predict system-level RUL. The proposed system-level stochastic approach was robust and accurate in RUL prediction but not computationally efficient when compared with inverseFORM. Sai Sarathi Vasan, Chen, \& Pecht (2013) proposed a model-based approach for system-level RUL prediction of the radio-frequency receiver system. The proposed model consists of three steps: knowledge-based creation, offline and on-line testing. In the knowledge-based creation step, system parameters and critical circuits were identified. Authors decomposed an electronic system into circuit components to detect only the critical ones to minimize the resources and complexity. In the off-line step, fault indicators and failure threshold were identified and finally, prognostics was performed by PF in an on-line step. Fault tree (FT) failure analysis technique which is widely used in safety analysis of aircraft systems, study interactions of sequences of sub-events with the top event. Daigle, Sankararaman, \& Roychoudhury (2016) presented a methodology based on FT technique for system-level RUL prediction by combining individually independent components RULs of aircraft environmental control system. Ferri et al. (2013) combined system architecture information with individual component RULs to obtain system RUL of the simplified electrical system.

Jie Liu \& Zio (2016) proposed a reliability assessment and system RUL prediction framework based on a recursive Bayesian method for the system with multiple dependent components. The system states were estimated by the recursive Bayesian method and Monte Carlo simulation was applied accurately in RUL prediction of nuclear power plants' residual heat removal system at system-level reliability.

Although, some papers on system-level PHM exist, there remain many issues and/or challenges to be solved to develop system-level PHM methodologies. Challenges regarding system-level PHM can be stated as, model structure and parameters uncertainties, nonlinearity of system model, environmental effects, measurement noise and component degradation interactions.

\section{UNCERTAINTY QUANTIFICATION}

Since fault prognostics methodologies deal with machine future health state prediction, there are many uncertainties parameters that influence the prediction accuracy. Sources of fault prognostics uncertainty can be categorized as following;

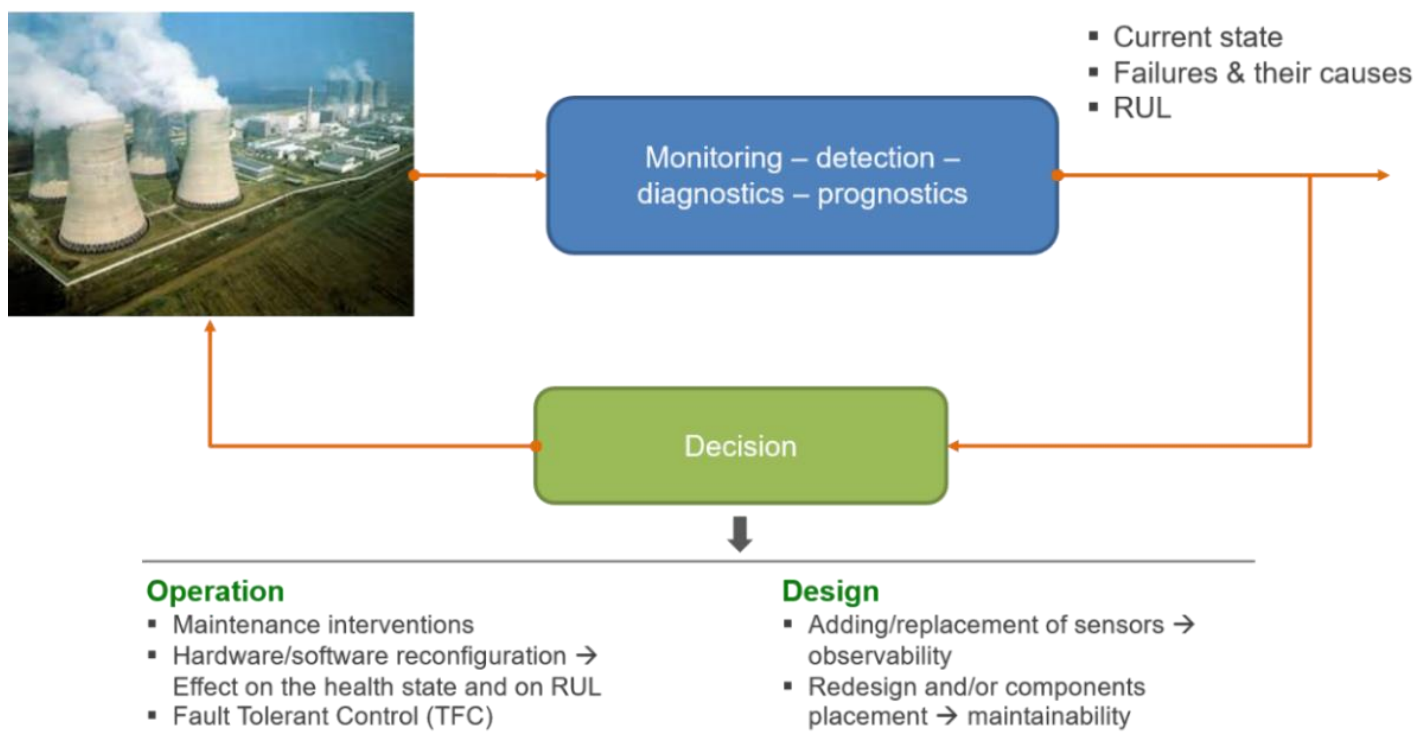

Fig. 9. Decision making. 


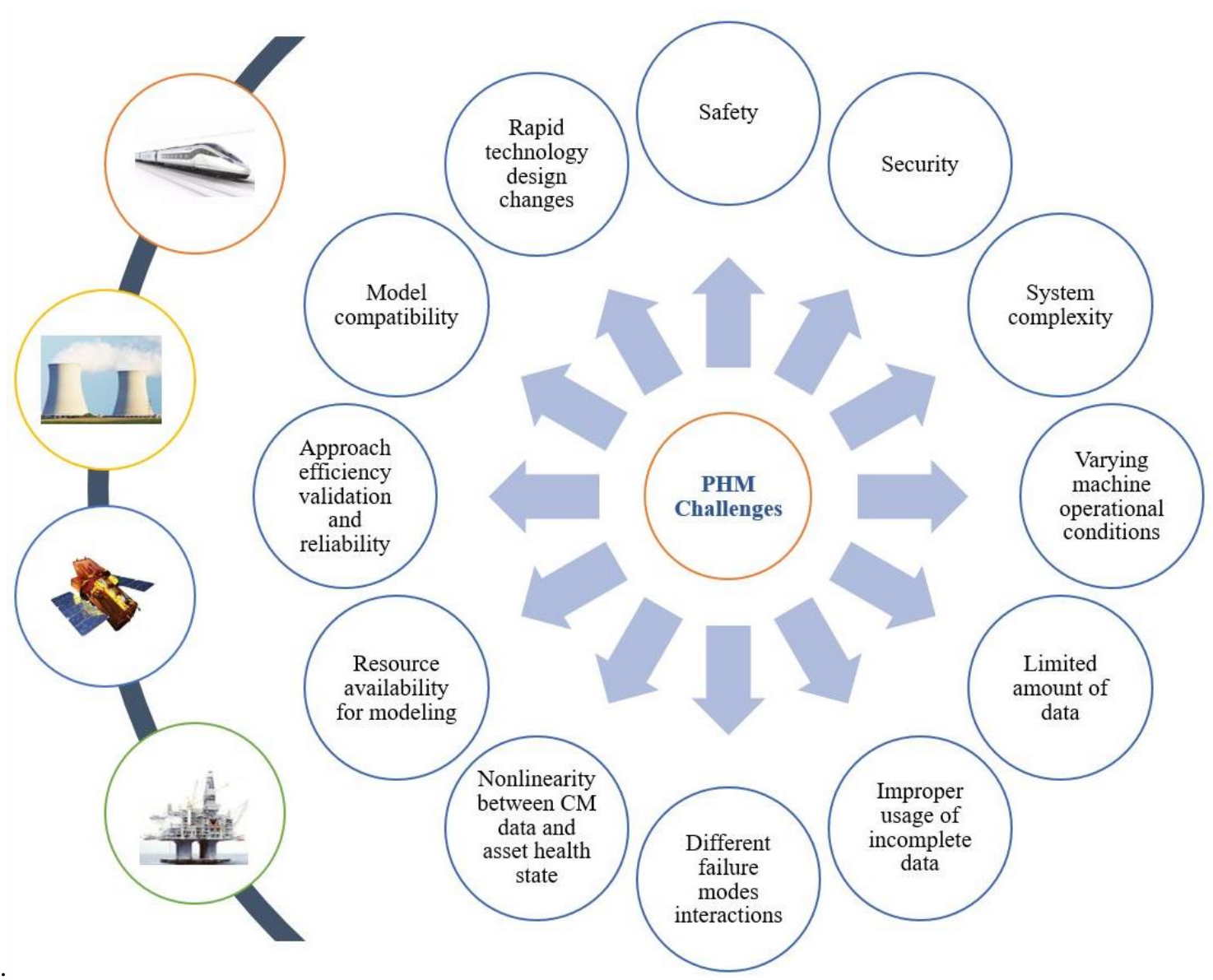

Fig. 10. PHM challenges.

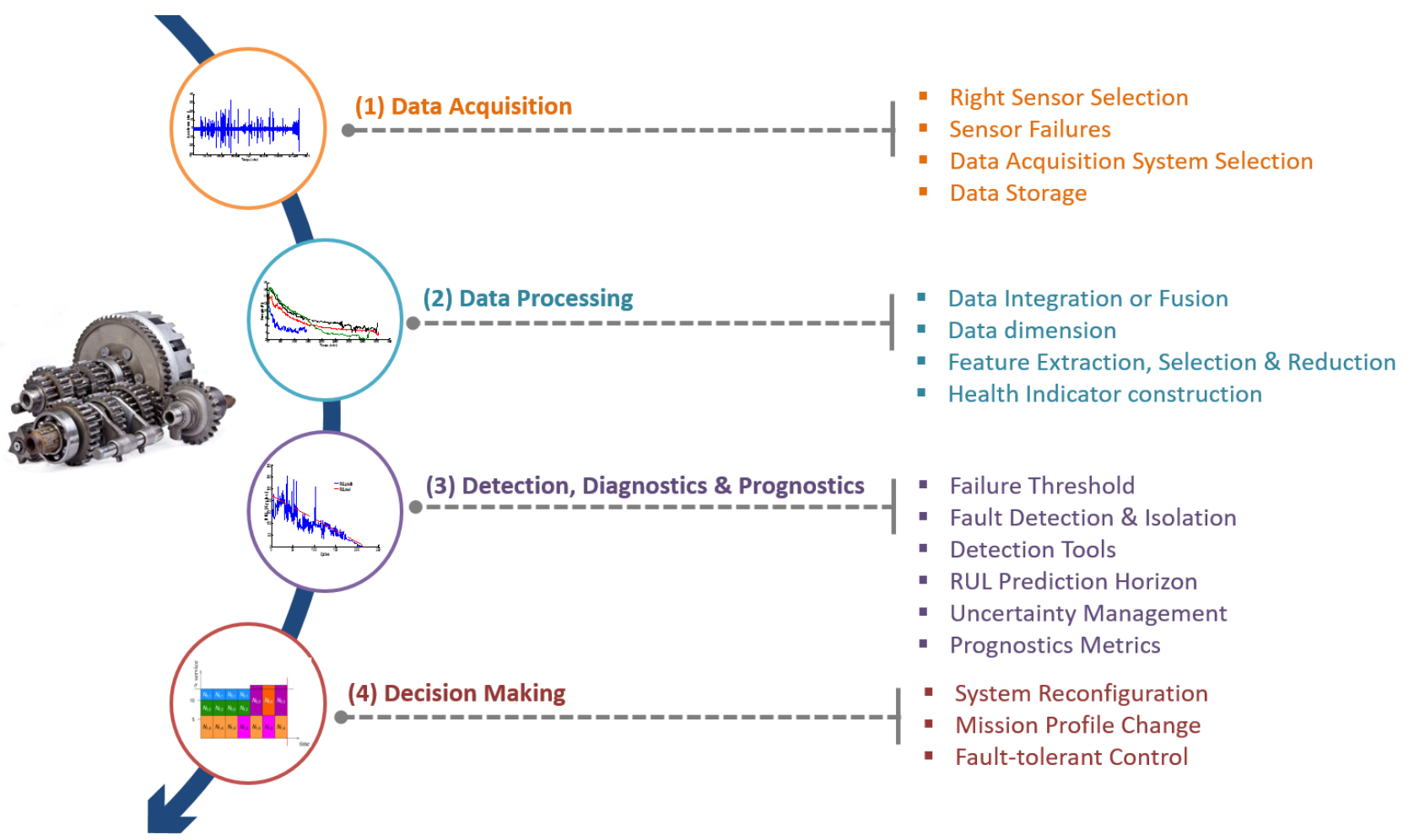

Fig. 11. Challenges faced in PHM steps. 
Uncertainty in system parameters: this concerns the uncertainty in the values of the physical parameters of the system (resistance, inductance, stiffness, capacitance, etc.). This uncertainty is induced by the environmental and operational conditions where the system evolves. This can be tackled by using adequate methods such interval ones.

Uncertainty in nominal system model: this concerns the imprecisions in the mathematical models which is generated to represent the behavior of the system. These imprecisions (or uncertainties) can be the result of a set of assumptions used during the modeling process and which lead to models that don't fit exactly the real behavior of the system. Fig. 12 illustrates an example for this type of uncertainty for Micro Electro Mechanical Systems (MEMS) (Skima, Medjaher, Varnier, Dedu, \& Bourgeois, 2016a) which degraded differently under the same operational profiles.

Uncertainty in system degradation model: the degradation model can be obtained from accelerated life tests which are conducted on different data samples of a component. In practice, the data obtained by accelerated life tests performed under the same operating conditions may have different degradation trend. This difference in the degradation trends can then be considered as an uncertainty in the degradation models derived from the data related to the accelerated life tests.

Uncertainty in prediction: uncertainty is inherent to any prediction process. Any nominal and/or degradation model predictions are inaccurate which is impacted by several uncertainties such as uncertainty in the model parameters, the environmental conditions and the future mission profiles. The prediction uncertainty can be tackled by using Bayesian and online estimation and prediction tools (e.g. $\mathrm{PF}$ and Kalman filter etc.).

Uncertainty in failure thresholds: the failure threshold is important in any fault detection and prediction methods. It determines the time at which the system fails and consequently the remaining useful life. In practice, the value of the failure threshold is not constant and can change in time. It can also change according to the nature of the system, operating conditions and in the environment which

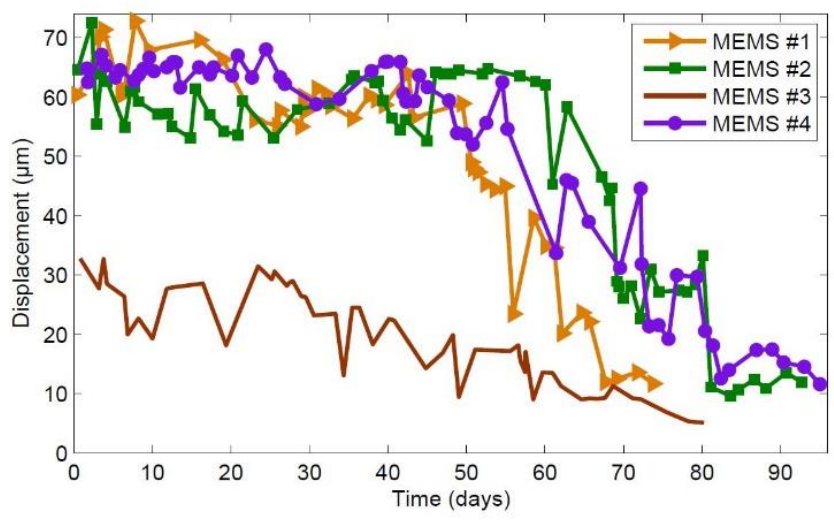

Fig. 12. Uncertainty in degradation model of MEMS (Skima et al., 2016a). it evolves. All these parameters induce uncertainty which should be considered in the definition of the failure threshold.

Sankararaman, (2015) analyzed the uncertainty in fault prognostics by quantifying the sources of uncertainty from different aspects of view. In (Sun, Zuo, Wang, \& Pecht, 2014), the authors developed state-space-based degradation model to reduce the crack growth prediction uncertainty based on data fusion. A similar work conducted in fault prognostics uncertainty quantification can be found in (Duong \& Raghavan, 2017; Sankararaman \& Goebel, 2013; Zhao, Tian, \& Zeng, 2013) articles. Hence, it's important to quantify the uncertainty sources in fault prognostics.

\section{PHM APPROACHES- A REVIEW}

In this section, we have reviewed scientific papers which have been published in component-level PHM, especially in complex components which have a high maintenance cost and severe failure consequences. And they were summarized under three categories, in general: modelbased (or physics-based), data-driven and hybrid approach with their drawbacks and merits. The Experience-based approaches, which is solely based on expert-knowledge, is not presented in this paper. Only the most studied approaches are presented and reviewed. PHM approaches are illustrated in Fig. 13 with their pros and cons.

\subsection{Model-based Prognostics Approaches}

In Model-based prognostics approaches, the behavior of a system/component degradation process leading to failure is described by mathematical models and/or equations derived from physical systems. Derived mathematical model is combined with condition monitoring data to identify model parameters, then used to predict the future evolution of component health state. Model-based approaches are more accurate than other approaches and have long-term RUL prediction horizon but need good expert knowledge. Deriving models from real physical systems is very challenging due to system complexity and stochastic degradation behavior of components. A modelbased approach for Li-ion cells is illustrated in Fig. 14. The equivalent battery circuit degradation model was adapted from Mohamed et al. (2010).

Commonly used model-based prognostics approaches in literature are Paris-Erdogan law (Irwin \& Paris, 1971; Paris \& Erdogan, 1963) and Forman law (Forman, 1972). In (LI, Kurfess, \& Liang, 2000), stochastic defectpropagation model was presented based on Paris equation for bearing defect prognostics.

Feasibility of proposed approach was tested by numerical simulations. Gearboxes are force transmission components of mechanical systems that need health assessment due to its importance. In (C. J. Li \& Lee, 2005), was proposed model-based methodology based on Paris crack propagation to predict RUL of cracked gears. Gear meshing stiffness was identified by embedded model from measured 


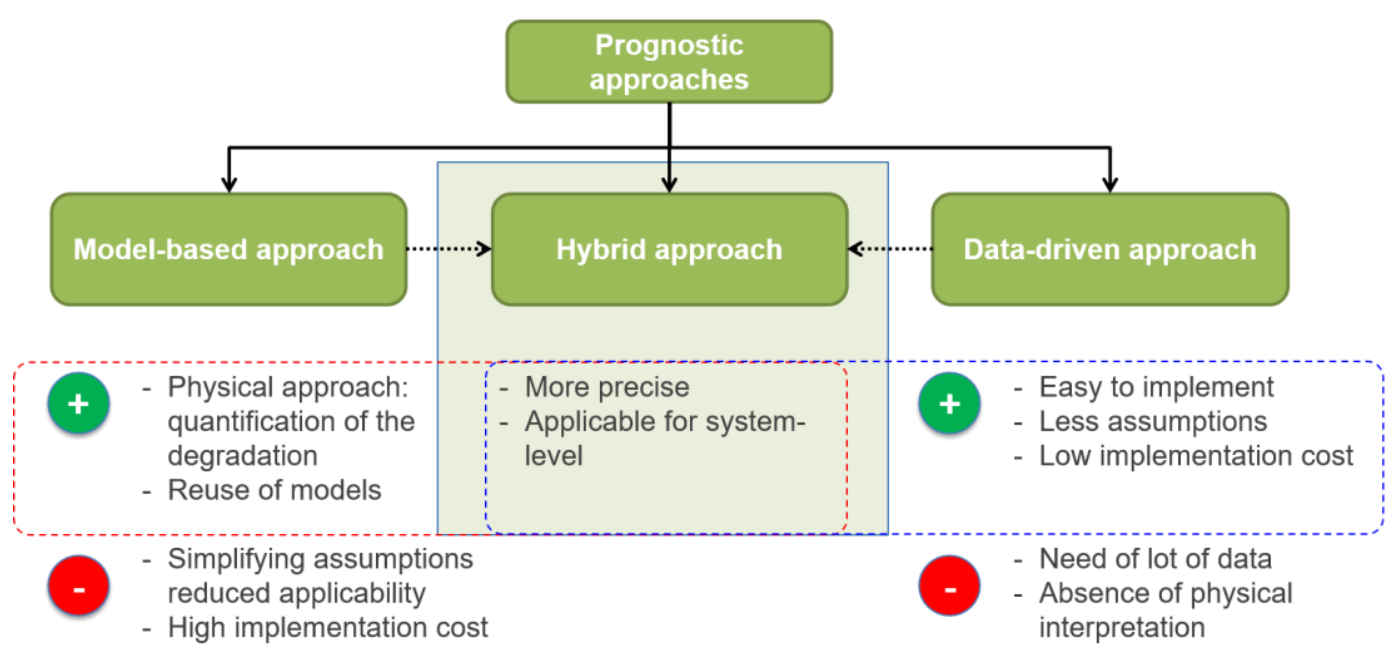

Fig. 13. PHM approaches.

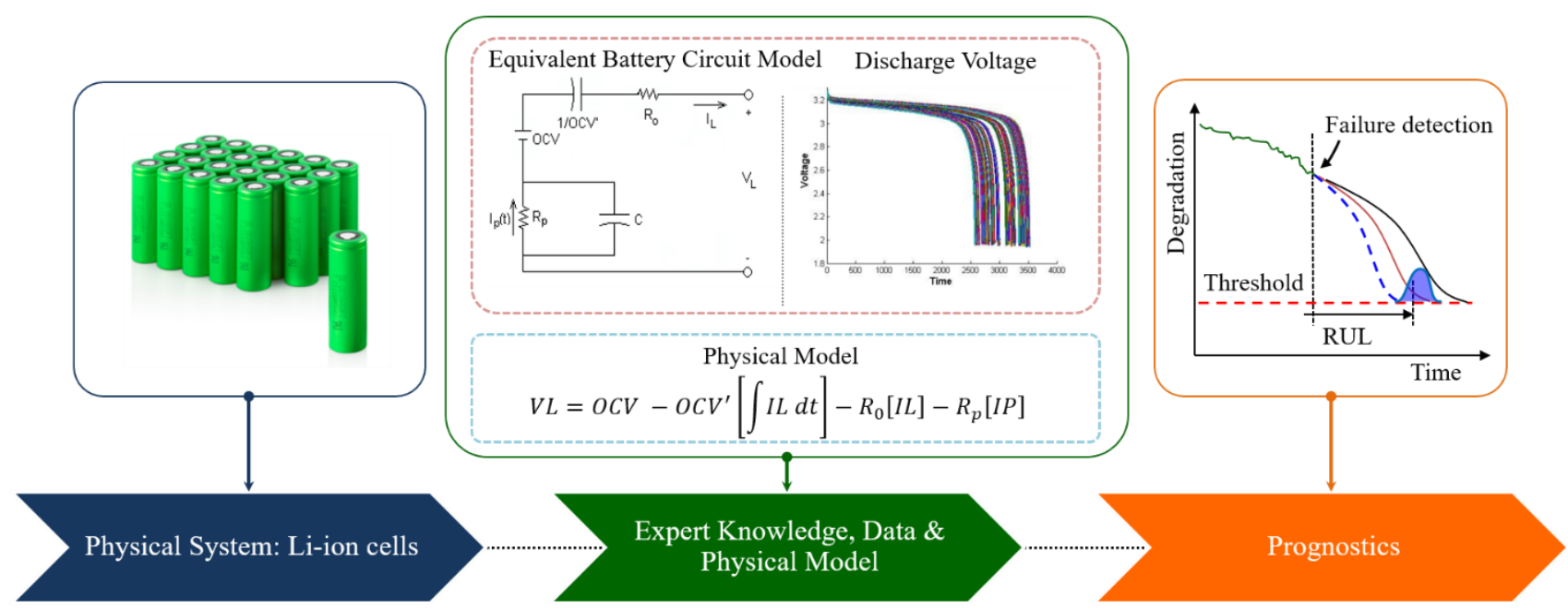

Fig. 14. Model-based prognostics approach for Li-ion battery.

vibration data. Experimental results showed that modelbased approach could accurately predict RUL of gear crack with minimum error. In (Marble \& Morton, 2006), was developed a model-based approach for turbine engine bearing spall progression prognostics. Depending on operating conditions, presented model could compute failure time and spall growth trajectories very well and prediction uncertainty was reduced by model updating. In (Choi \& Liu, 2007), was studied spall progression life model due to crack formation and wear of rolling contact. Crack initiation and propagation models were combined with developed spall progression model to predict finish hard machined specimens' total life. Developed spall progression life model was experimentally tested and was less sensitive to different machine operations when compared with fatigue life models.

Cracks in mechanical components of complex machines can cause serious failures in the industry unless diagnosed well. In (Pennacchi \& Vania, 2008), was presented modelbased diagnostics approach for failure identification in gas turbines. They analyzed shaft vibrations caused by crack propagation during load coupling of gas turbines and successfully obtained good diagnostics results to avoid catastrophic failures.

In many safety-critic complex systems, such as bridges ( $\mathrm{J}$ $\mathrm{He}, \mathrm{Lu}$, \& Liu, 2012) and aircraft (Campbell \& Lahey, 1984), fatigue crack growth prognostics is efficiently used for structural health management to avoid catastrophic failures. In (Guan et al. 2012), was proposed Maximum relative Entropy (MrE) to manage measurement uncertainties. Physics of fatigue crack was modeled using Paris-Erdogan law (Paris \& Erdogan, 1963) and was validated on using Virkler (Virkler, Hillberry, \& Goel, 1979) fatigue crack propagation dataset. In comparison with the classical Bayesian method, proposed approach gave improved prediction results in fatigue crack prognostics.

Self-adaptive RUL prediction methodology based on Paris law for rolling element bearing prognostics was studied in 
(Liang et al., 2014) paper, using vibration data acquired by accelerated aging procedure and bearing defect propagation was effectively predicted without a priori knowledge about model parameters.

Kalman Filter (KF) (Kalman, 1960), which is a widely known Bayesian method, has been used to model linear systems with additive Gaussian noise for estimation and prediction purposes. In (Lim \& Mba, 2015), Switching Kalman Filter (SKF) was adopted for model estimation and RUL prediction of aircraft gearbox bearings using CM data. And it was concluded that, when CM data is available, using SKF for diagnostics and prognostics of bearing degradation, could support to make better maintenance decisions. Prognostics of Proton Exchange Membrane Fuel Cells (PEMFC) were studied in (Bressel et al. 2016). Extended Kalman Filter (EKF) was used in State of Health (SoH) estimation and RUL prediction of fuel cells and it was stated that EKF could offer good $\mathrm{SoH}$ estimation and accurate RUL prediction results using experimental data for degradation modeling. In (Andre et al. 2013), was proposed an online mathematical approach based on a combination of dual KF and Support Vector Machines (SVM) to estimate State of Charge (SoC) and SoH values of Li-ion batteries. The developed approach was validated using internal resistance features from real driving cycle test data which gave accurate and comprehensive results for both $\mathrm{SoH}$ and SoC estimation of aged Li-ion batteries in a laboratory environment. Since batteries are electrochemical substances, they have nonlinear degradation pattern due to many environmental effects. In (Burgess, 2009), was developed a methodology using KF to estimate remaining service life of the nonlinearly degraded lead-acid battery. Nonlinear capacity degradation of the lead-acid battery was modeled by dividing capacity time series data into two phases; phase-1which was assumed as healthy part and phase- 2 which as the faulty part where degradation is more rapid. KF algorithm was conducted when capacity degradation reached to phase- 2 in order to predict the remaining service life of the leadacid battery. An important issue that was not handled in this paper is how to detect incipient failure from nonlinear capacity degradation time series before jumping to phase2. The uncertainty of degradation parameters due to environmental effects is one of the main problems in $\mathrm{Li}$-ion SoC and SoH estimations.

Particle filters (PF), which are known as state-space models, has been used in many prediction problems. Jouin et al. (2014) studied PEMFC stack degradation and developed prognostics model for RUL estimation using PF. Despite the PF's robustness in prediction process, it has a degeneracy phenomenon. After some iteration, particle weights tend to zero affecting estimation result. This problem was handled in (H. Dong et al 2014) by adopting SVM to resample weights in order to avoid degeneracy problem. Internal resistance and capacity features were used as system health indicators to estimate $\mathrm{SoH}$ of $\mathrm{Li}$-ion batteries. Integration of SVM-PF algorithm improved SoH estimation and RUL prediction when compared with standard PF itself. Piyush Tagade et al. (2016), developed an improved $\mathrm{PF}$ algorithm to indicate battery state estimation at different driving cycle protocols and was compared with KF algorithm. Proposed model accurately predicted battery parameters at different driving cycle protocols outperforming KF algorithm. Filtration, which is a process of cleaning and/or trapping suspended particles from a liquid, is an important process in many engineering applications, such as automotive, chemical industry, nuclear plants, etc. In (Omer F. Eker, Camci, \& Jennions, 2015), was presented a physics-based model for filter clogging phenomena. PF was integrated with presented physics model to estimate RUL of fuel filters and it was validated on experimental data acquired from test rig under laboratory environment. Zio \& Peloni, (2011) presented PF-based prognostics framework for dynamic fatigue crack propagation. A tutorial on PF with Matlab code implementation was presented in (An, Choi, \& Kim, 2013). Marine J. et al. (2016) presented comprehensive review.

Even though model-based approaches have accurate prognostics results, they might not be a feasible solution in many industrial applications, due to failure type uniqueness, dynamic damage evolution and specific theoretical knowledge about the system. Failure progression of components and/or subsystems in a complex system might be different from each other which limits the applicability of physical models in system level prognostics. Model-based prognostics tools studied in the literature, are given in Table 2 below with their application areas, advantages, and disadvantages.

\subsection{Data-driven Prognostics Approaches}

Data-driven approaches attempt to build degradation models using information from $\mathrm{CM}$ data collected via installed sensors and to predict future health state instead of building physical models. Data-driven approaches can be easily applied in prediction problems and require more computational time than model-based approaches. The accuracy of data-driven approaches is highly dependent on the amount of CM data available. Data-driven prognostics approach is illustrated in Fig. 15. And battery degradation model in this present figure was adapted from the work of W. He et al. (2011).

In traditional prognostics methodologies, results of multiple algorithms are compared and the one which gives the best result is selected for prediction of system health state while discarding other algorithms. Ensemble based prognostics approach which combines multiple algorithms using different weighted-sum functions for accurate RUL prediction was proposed to overcome this limitation in $\mathrm{CH}$ Y. et al. 2012). Proposed ensemble approach was tested using 2008 IEEE PHM challenge problem: power transformer and electric cooling fan problems, by combining 5 different prognostics algorithm which increased RUL prediction accuracy.

ARIMA, which is the generalization of ARMA, is another widely-used approach in time series forecasting aiming to describe autocorrelation of the data. In (Wei Wu, 2007), was proposed an improved ARIMA model for rotating 


\begin{tabular}{|c|c|c|c|}
\hline Prognostics Tools & Advantages & Disadvantages & Application \\
\hline Paris' law (PL) & $\begin{array}{l}\text {-Model parameters are } \\
\text { adaptable for conditional } \\
\text { changes }\end{array}$ & $\begin{array}{l}\text {-Linear correlation with } \\
\text { defect size and vibration } \\
\text { RMS level } \\
\text {-Empirical determination of } \\
\text { material constants is needed }\end{array}$ & $\begin{array}{l}\text {-Bearings, gearbox, fatigue } \\
\text { crack propagation }\end{array}$ \\
\hline Forman law (FL) & $\begin{array}{l}\text {-Links both monitored data and } \\
\text { crack growth physics to life } \\
\text { models }\end{array}$ & $\begin{array}{l}\text { - Poor results for complex } \\
\text { systems }\end{array}$ & -Fatigue crack propagation \\
\hline $\begin{array}{l}\text { Fatigue spall } \\
\text { initiation/progression model } \\
\text { (FSI/P) }\end{array}$ & $\begin{array}{l}\text {-Calculates time up to initiation } \\
\text { and from initiation up to failure } \\
\text {-Damage is cumulative }\end{array}$ & $\begin{array}{l}\text {-Many parameters to be } \\
\text { determined }\end{array}$ & $\begin{array}{l}\text { - Bearings, fatigue crack } \\
\text { propagation }\end{array}$ \\
\hline Kalman Filter (KF) & $\begin{array}{l}\text {-Estimates current/future states } \\
\text {-Estimation error is corrected } \\
\text { with the latest measurement }\end{array}$ & $\begin{array}{l}\text {-System/measurement model } \\
\text { need to be defined } \\
\text {-Sensitive to noise } \\
\text {-Applicable to linear systems } \\
\text { with Gaussian noise }\end{array}$ & $\begin{array}{l}\text { - Gearbox bearings, PEMFC, } \\
\text { batteries }\end{array}$ \\
\hline Particle Filter (PF) & $\begin{array}{l}\text {-Applicable to non-linear } \\
\text { systems with non-Gaussian } \\
\text { noise } \\
\text {-Better accuracy } \\
\text {-Avoids degeneracy problem by } \\
\text { resampling }\end{array}$ & $\begin{array}{l}\text {-System/measurement model } \\
\text { need to be defined } \\
\text {-High dimensional data } \\
\text { increases computational cost }\end{array}$ & $\begin{array}{llr}\text {-PEMFC, } & \text { batteries, } & \text { filter } \\
\text { clogging, } & \text { fatigue } & \text { crack } \\
\text { propagation } & & \\
& & \end{array}$ \\
\hline
\end{tabular}

Table 2. Model-based prognostics tools and their advantages and disadvantages.

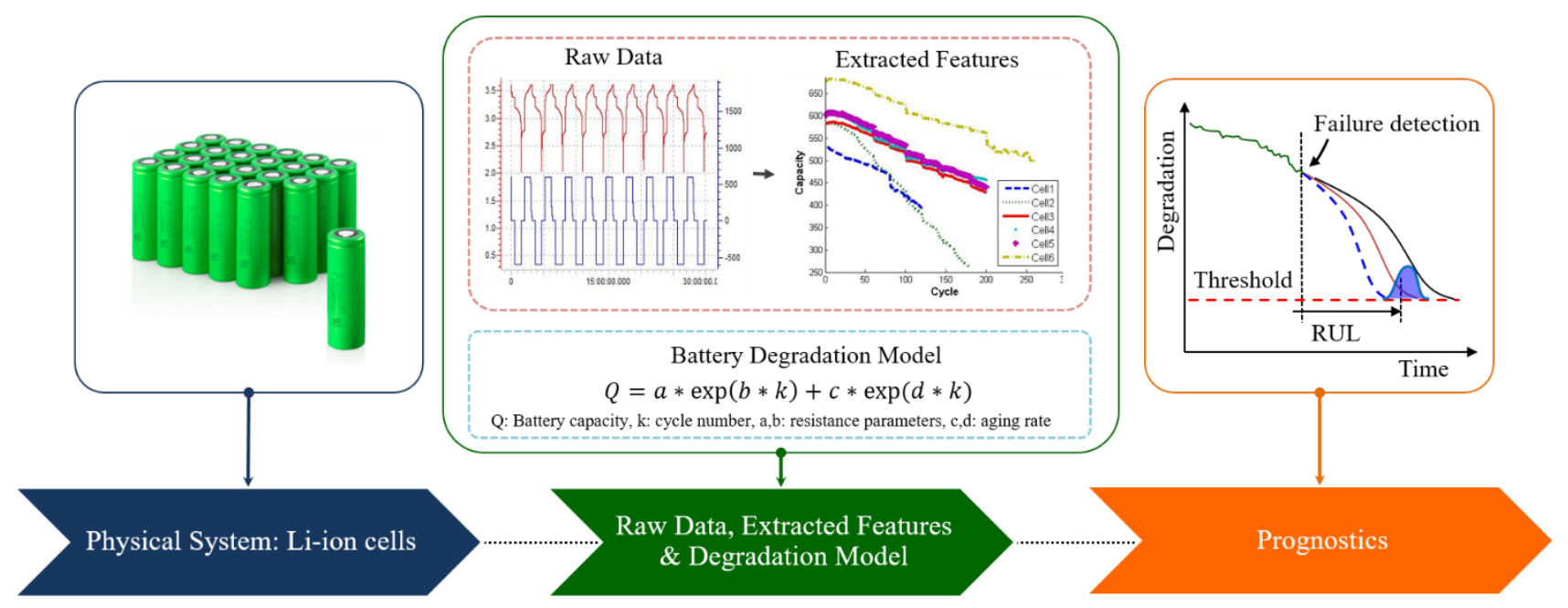

Fig. 15. Data-driven prognostics approach for Li-ion battery.

machinery prognostics which could detect underlying changes from machine degradation data. It was validated on vibration signals collected from test rig and gave better prediction result than standard ARIMA. Furthermore, prognostics on miniature bulb degradation data was studied by modeling trend and seasonal components of time series analysis technique in (T. Huang et al. 2010).

Jianbo L. et al. (2007) developed a novel prediction algorithm so-called Match Matrix (MM) with long prediction horizon. MM was formed by comparing two degradation data based on similarity distances. If the similarity or match value between currently developing degradation and historical one is bigger, then one can use historical degradation time series data in modeling to predict future behavior of currently developing degradation. MM prediction results were compared with Elman Recurrent Neural Network (ERNN) and gave better long term prediction accuracy. Gaussian Process Regression (RPG) statistical non-parametric method was adopted for nuclear plant prognostics in (Piero Baraldi, Mangili, \& Zio, 2015).

GPR was validated on simulated creep growth time series and real filter clogging degradation with accurate RUL prediction results. In (Yu, 2011), Gaussian Mixture (GM) was integrated with feature extraction methodology for bearing defect diagnostics. In (P. Wang, Youn, \& $\mathrm{Hu}$, 2012), an offline-online methodology for structural health prognostics was proposed. In online step, the probabilistic recursive Bayesian algorithm was used for learning system health level whereas in offline step similarity-based 
interpolation was used to predict the lifetime of an electric cooling fan.

An offline-online methodology for prognostics of $\mathrm{CNC}$ machines using Bayesian network was proposed in (D. A. Tobon-Mejia, Medjaher, \& Zerhouni, 2012). In an offline phase, reliable features were extracted from raw data and were fed for further modeling using Mixture of Gaussian Hidden Markov Model (MoG-HMM), whereas, in the online phase, the learned model was used in cutting tool health assessment and RUL estimation processes. Wavelet packet decomposition was adopted in feature extraction using vibration signals and MoG-HMM was used to model bearing degradation and RUL estimation in (Diego A. et al., 2012). In (M. Dong \& He, 2007), was proposed diagnostics and prognostics framework for hydraulic pump health monitoring based on hidden semi-Markov Models (HSMM). Furthermore, authors modified the forwardbackward algorithm to estimate model parameters and using HSMM estimated time duration of hidden states performed RUL prediction which was better than traditional HMM. Research done in battery prognostics mainly aims to estimate State-of-Charge ( $\mathrm{SoH})$, State-ofHealth (SoH) and to predict RUL. Two prognostics methods; linear and artificial neural network (ANN), were implemented to predict RUL of batteries using capacity degradation time series in (S. Lee et al, 2012). ANN approach performed better than linear model since it gives better results in one-step-ahead RUL predictions. Li-ion self-recovery which is known as cell regeneration phenomenon was investigated and combined with capacity degradation data for prognostics of batteries utilizing a combination of GPR functions in (D. Liu et al., 2013). As a result presented approach reduced prediction uncertainty while increasing accuracy. Prognostics of Li-ion batteries based on capacity degradation time series was presented using Autoregressive (AR) model with Particle Swarm Optimization (PSO) algorithm in (Long, Xian, Jiang, \& Liu, 2013) paper for PF.

Reliability, availability and safety are subjects of great importance in many complex systems and indeed in the railway industry. Railway turnout systems, which are electro-mechanical structures, are key contributors to the reliability and availability of a railway network. In (Omer Faruk Eker et al., 2011), the authors developed a prognostics methodology to detect and forecast failures in electro-mechanical systems named as simple-state based prognostics method. Results of proposed methodology were quite good in comparison with Hidden Markov Models (HMM) using real datasets collected from a railway turnout machine. Time Delay Neural Network (TDNN) was used for failure prediction using real sensory data collected from a railway turnout machine in (Halis Yilboga, Ömer Faruk Eker, 2010). Data-driven prognostics approaches build degradation models using monitored data collected via installed sensors and they may be more practical and/or available solution for diagnostics and prognostics of complex systems in many applications instead of building physical models. However, those models have no or less physical meaning which makes them less sensitive to real system behavior. Table 3 presents application areas, advantages, and disadvantages of data-driven prognostics tools.

\begin{tabular}{|c|c|c|c|}
\hline Prognostics Tools & Advantages & Disadvantages & Application \\
\hline ARIMA Models & $\begin{array}{l}\text {-Applicable to linear systems with } \\
\text { stationary behavior } \\
\text {-Uses less amount of data }\end{array}$ & $\begin{array}{l}\text {-Short term prediction } \\
\text {-Not useful for non-stationary } \\
\text { processes }\end{array}$ & - Rotating machinery \\
\hline Match Matrix (MM) & $\begin{array}{l}\text {-Deals with high dimensional data } \\
\text {-Provides long term prediction } \\
\text {-Suitable for non-stationary } \\
\text { processes }\end{array}$ & $\begin{array}{l}\text {-Needs sufficient historical data } \\
\text {-Data should have degradation } \\
\text { trend }\end{array}$ & - Rotating machinery \\
\hline $\begin{array}{l}\text { Gaussian Mixture } \\
(\mathrm{GM})\end{array}$ & $\begin{array}{l}\text {-Many Gaussian functions can be } \\
\text { used to approximate an arbitrary } \\
\text { distribution and accuracy }\end{array}$ & $\begin{array}{l}\text {-Initialization methods are } \\
\text { important in parameter } \\
\text { optimization } \\
\text {-Determining number of mixtures is } \\
\text { difficult }\end{array}$ & - Bearings, CNC machines \\
\hline $\begin{array}{l}\text { Gaussian Process } \\
\text { Regression (GPR) }\end{array}$ & $\begin{array}{l}\text {-Adaptable to environment and can } \\
\text { learn from experience }\end{array}$ & $\begin{array}{l}\text {-Needs covariance function } \\
\text { determination } \\
\text {-Suitable for Gaussian likely hood }\end{array}$ & $\begin{array}{l}\text { - Nuclear power plants, } \\
\text { batteries }\end{array}$ \\
\hline $\begin{array}{l}\text { Artificial Neural } \\
\text { Networks (ANN) }\end{array}$ & $\begin{array}{l}\text {-Applicable for complex systems and } \\
\text { which have non-linear behavior } \\
\text {-Adaptable to the system }\end{array}$ & $\begin{array}{l}\text {-Network structure is not } \\
\text { determinable } \\
\text {-Needs resources for computation }\end{array}$ & $\begin{array}{l}\text { - Bearings, batteries, } \\
\text { turnout point machines }\end{array}$ \\
\hline Fuzzy Logic (FL) & $\begin{array}{l}\text {-Inputs can be imprecise } \\
\text { noisy/incomplete } \\
\text {-Appropriate for complex systems }\end{array}$ & $\begin{array}{l}\text {-Needs rule development based on } \\
\text { expert knowledge }\end{array}$ & - Bearings \\
\hline $\begin{array}{l}\text { Bayesian Networks } \\
\text { (BN) }\end{array}$ & $\begin{array}{l}\text {-The number of structure parameters } \\
\text { are reduced by conditional } \\
\text { probability distribution } \\
\text {-Visualizes variable pair dependency } \\
\text { links }\end{array}$ & $\begin{array}{l}\text {-Has complex and costly learning } \\
\text {-Prior knowledge is needed }\end{array}$ & - Bearings \\
\hline
\end{tabular}

Table 3. Data-driven prognostics tools and their advantages and disadvantages. 


\subsection{Hybrid Prognostics Approach}

Both model-based and data-driven prognostics approaches have their own merits and limitations as was discussed in previous sections. The hybrid prognostics approach, which is a newly developing PHM approach, aims to integrate merits of different approaches while minimizing limitations for better system and/or component level health state estimation and RUL prediction. None of the prognostics approaches that is presented in the literature is superior to another. Hence, it's important to note that superiority of prognostics approaches can only be case specific.

In (Liao \& Köttig, 2014), was presented a comprehensive literature review aiming to developed a hybrid prognostics approach combining advantages and disadvantages of different prognostics approaches. Authors validated developed a hybrid approach for Li-ion degradation as a case study to show potential benefits. They categorized prognostics models as experience-based, data-driven, and physics-based models and presented 5 different hybrid approach classes as illustrated in Fig. 16. Here we briefly explain those classifications with their advantages and disadvantages. Interested readers are kindly referred to the original paper (Liao \& Köttig, 2014) for more information.

Class 1 - combines Experience-based and Data-driven approach models. Experience-based approaches, which is solely based on expert-knowledge, is integrated with datadriven models for asset prognostics. The advantage of this hybrid approach is that expert-knowledge can help to determine system faults and those rules can be used in datadriven models for RUL prediction. However, expertknowledge may not capture all faults occurring in system degradation and this hybrid approach is not a feasible option for long-term RUL predictions. Satish \& Sarma (2005), presented a cost-effective method for bearing fault prognostics combining ANN and fuzzy logic. Results of developed hybrid approach were more suitable for bearing health assessment than ANN.

Class 2-combines Experience-based and Physics-based approach. This hybrid approach integrates expertknowledge to improve the physics-based model. This is similar to Class 1, the only difference is, it uses physicsbased models for prognostics. In (Swanson, 2001), was presented a hybrid approach for crack propagation problem using KF and fuzzy logic algorithms. KF was used to detect feature changes and estimate remaining time to failure where fuzzy logic was adopted to define failure thresholds for vibration signals of machines under different operational modes.

Class 3 - combines multiple Data-driven approach models. In this hybrid approach, two or more data-driven models are combined to improve asset performance prediction by means of information fusion. In this class, one data-driven approach can be used to estimate the health state and a second one to predict asset residual life. In (Gebraeel et al., 2004), was developed a hybrid approach for bearing failure prediction based on ANN. Authors collected vibration signals from 25 accelerated bearing tests and trained $25 \mathrm{ANN}$ for bearing failure time prediction. Then residual lifetime prediction was accomplished by weighting the outputs of ANNs. In (Peng \& Dong, 2011), was proposed a hybrid approach for pump wear prognostics. Grey prediction model which also was studied in (Gu et al., 2010) for electronic prognostics was integrated with aging factor integrated HMM RUL prediction algorithm for asset prognostics. Only the drawback of this approach is RUL prediction process that is carried out by data fusion, could be computationally hard if multiple models are used.

Class 4 - combines Data-driven and Physics-based approach models. This hybrid approach aims to integrate the strengths of both data-driven and physics-based approaches for accurate performance prediction. There are many research papers which have been studied in the literature falling into this class, only some of them are presented here.

Kumar et al. (2008) proposed hybrid approach utilizing data-driven approach and model-based approach for electronic products prognostics. Hong-feng (2012), presented general fusion framework utilizing both merits of data-driven and model-based approaches to increase system reliability and to reduce maintenance cost. In many complex assets, performing online health measurement may not be practical and even impossible and thus modelbased approach such as PF is not applicable for machine prognostics. P. Baraldi et al. ( 2013) proposed a novel ensemble approach embedding ANN in the PF scheme for crack depth prediction problem. The main idea for this approach proposition is based on unavailability of measurement model but only dataset itself. Different datasets were generated to train different ANNs and their

Hybrid Prognostics Approaches Categorization

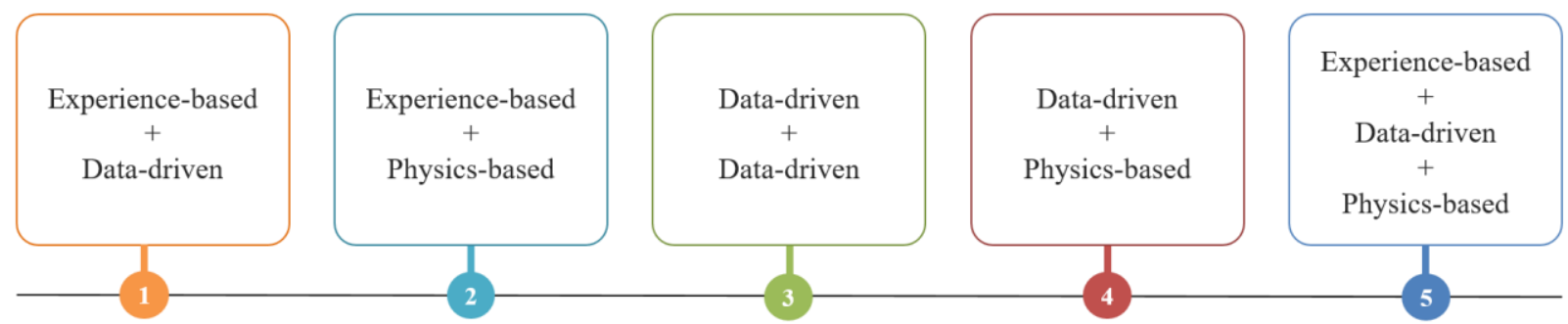

Fig. 16. Hybrid prognostics approaches (modified from (Liao \& Köttig, 2014)). 
outputs were combined to get ensemble output. It was validated on fatigue crack propagation dataset and results showed that if ANN is trained with large dataset it was possible to get accurate estimations. In (Goebel, Eklund, \& Bonanni, 2006), was presented a fusion prognostics approach combining data-driven and physics-based prediction results for bearing prognostics to reduce prediction uncertainty. The physics-based approach was based on a spall-growth model where the data-driven model was based on load and speed measurements from bearing spall growth. Results of both different approaches were fused by well-known Dempster-Shafer regression. Yang et al. (2016) presented a hybrid approach that integrates relevance vector machine (RVM) with a modelbased model for Li-ion capacity degradation. Several batteries were aged under aging process and capacity features were extracted for RUL prediction. Combined two exponential physical model was fit to capacity features to track degradation trend. Furthermore, both selective kernel ensemble-RVM and an exponential regression model were used to predict RUL of aged batteries and proposed hybrid approach prediction was pretty accurate when it was compared with ANN and SVM. In (Liao \& Köttig, 2016), was proposed a hybrid approach for Li-ion prognostics. They integrated two data-driven models and one physical model to reduce the uncertainty. Support vector regression was adopted to estimate measurement model and similarity-based prediction method in measurement prediction part. The output of second data-driven model was fed to PF algorithm for long-term Li-ion RUL prediction. They extracted internal resistance degradation features and used in hybrid model validation. It was concluded that prediction uncertainty and accuracy was better than traditional PF and data-driven method. Skima et al. (2016) presented a hybrid approach for Micro Electro Mechanical Systems (MEMS) prognostics. An approach involves two phases; online and offline. In offline phase degradation model was constructed based on health indices (HI) extracted from experimental data. The combination of two exponential regression model was constructed to represent MEMS degradation phenomena. Furthermore, in an online phase, a model-based method which is PF was adopted. Online phase also had learning stage and prediction stage. In learning stage, $\mathrm{PF}$ parameters were estimated and in prediction stage, RUL prediction was carried out. They claimed that proposed hybrid approach could predict RUL of MEMS effectively. The authors in (Pillai, Kaushik, Bhavikatti, Roy, \& Kumar, 2016) proposed a hybrid approach for gas turbine failure prognostics based on machine learning.

Class5 - combines Experience-based, Data-driven and Physics-based approach models. This class of hybrid approach fuses the strength of experience-based, datadriven and physics-based models. In (Orsagh, Sheldon, \& Klenke, 2003), was proposed a hybrid approach that fuses data-driven, physics-based prediction results with expertknowledge information to predict RUL of bearings. This category of the hybrid approach is not practical and hard to implement in real applications due to designing appropriate data fusion methodology to combine different types of information, resulting from different prognostics approaches.

In general, hybrid prognostics approach is a combination of heterogeneous models. It leverages advantages of different approaches to making robust RUL predictions. But the main challenge in hybrid approach is uncertainty management issue (Liao \& Köttig, 2014), which needs further improvements.

\section{RaIlway Industry - A CaSe Study on Bogie Diagnostics and Prognostics}

Reliability, availability and safety are subjects of great importance in many complex systems and indeed for the railway industry. When we compare railway vehicles with other industrial structures such as wind turbines and nuclear plants one can see a big difference between operating conditions. Wind turbines and nuclear plants are stationary where rail vehicles are mobile complex systems. Hence, maintenance and reliability policies can be slightly different than stationary complex systems for rail vehicles. Reliability-critical systems such as rolling stock may have longer life expectancy than other systems, which makes introducing new technology costly and difficult. To provide high safety and good comfort for passengers in rolling stock industry, wheel/rail contact, bogie design and component health assessment is very crucial especially for high-speed operations. Maintenance and online monitoring system platforms should be designed in such manner as to maintain train operations under different environmental conditions by optimizing maintenance to reduce costs and increase the fleet availability.

The Bogie, which is the most important and complex part of the rolling stock, is responsible for fundamental functions such as supporting car body, transferring brake and traction forces, train stability at high speeds, safe rail curve passing, good and comfort ride. Main bogie components are as follows: bogie frame, wheelset, primary and secondary suspension systems. Bogie and its components are illustrated in Fig. 17.

- Bogie frame is the main and largest structural component of the rail vehicle.

- Wheelsets are a complex structural component of bogie which involves axles, brake systems, and wheels.

- Primary suspension is the structural system that links wheelset to the bogie frame.

- The secondary suspension is the system which links bogie frame to the car body.

\subsection{Failure Mode, Mechanism and Effect Analysis (FMMEA)}

To develop an effective and robust diagnostics and prognostics system for bogie condition monitoring, one should have a comprehensive understanding of the component degradation behaviors and mechanisms under different load or environmental conditions. Bogie 


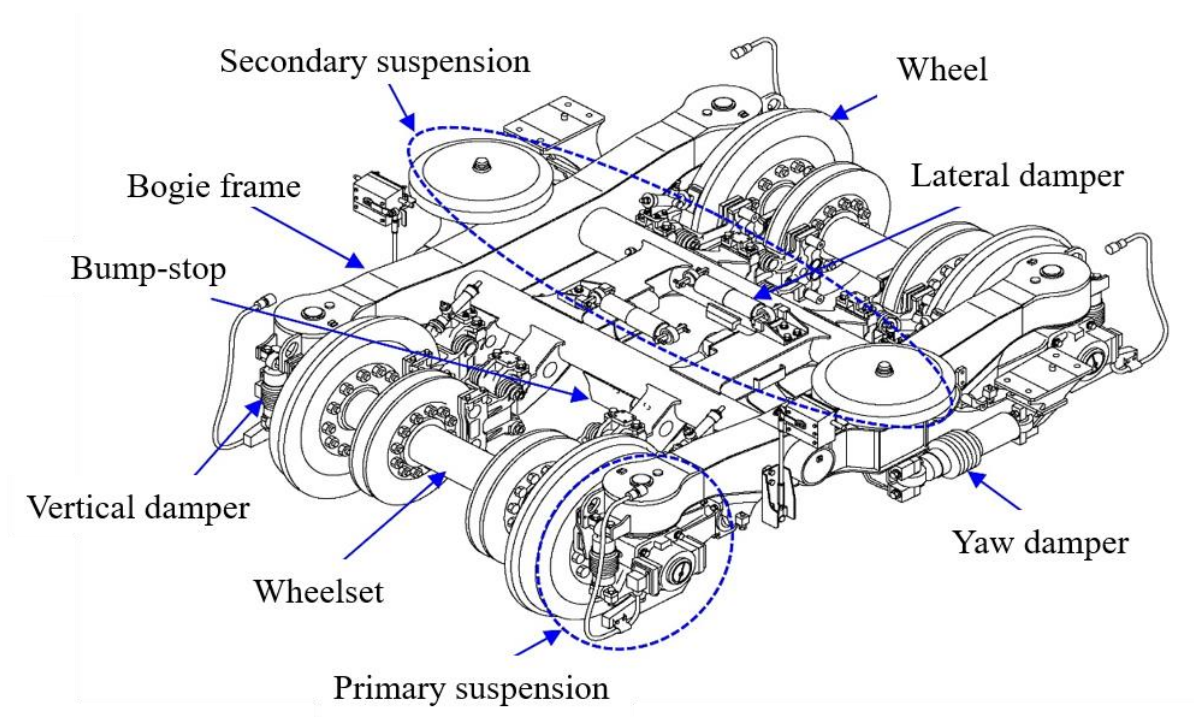

Fig. 17. Bogie components (adopted from (Ling et al., 2014)).

components are exposed to different stress levels resulting in component fatigue or wear. Such failures can lead railway industry to costly shutdowns even may have human causalities. To avoid or minimize component failures, we should understand failure modes and causes to perform better diagnostics and prognostics. Failure mode, mechanism, and effect analysis (FMMEA) is an efficient tool used to analyze component failures, identify the main causes or mechanisms and failure effects on the system and/or component operation (Hendricks, Williard, Mathew, \& Pecht, 2015; Mathew, Alam, \& Pecht, 2012). FMMEA can be used at the system level, subsystem, and component level. Failure mode describes the way of how physical failure occurs. Failure mechanism, on the other hand, describes the fundamental causes or processes such as chemical, physical and mechanical stresses that lead to a failure mode. Finally, the failure effect describes consequences or impacts of failures on the system and/or component operation. In Table 4, we have summarized some failure modes and mechanisms for the following bogie components: bogie frame, wheels, axle bearings, axle box, suspension (primary and secondary) system and gear box. Since bogies are critical components, diagnostics, and prognostics policies should be carefully carried out to avoid bogie failures and prevent rail vehicle from catastrophic disasters. The scientific papers conducted for fault diagnostics and prognostics of bogie components are as follows: Shahidi et al. (2014) presented vibration signal based condition monitoring strategy to estimate bogie component deterioration to analyze bogie hunting and reviewed critical criteria affecting the performance of bogie. To increase the efficiency of monitoring strategy authors addressed monitoring algorithms nearby sensing techniques. In (Hong et al., 2014), was studied bogie frame health monitoring by deploying online structural health monitoring (SHM) and diagnostics system to high-speed trains. A huge amount of signals was acquired during different operational profiles. Furthermore, different features were extracted from those signals to form damage indices to detect bogie frame damages. They concluded that online SHM diagnostics system could detect any kind of damages that might occur in bogie frame and could be used efficiently in real-world applications. Similar work was also studied in (Q. Wang, Su, \& Hong, 2014) to develop online SHM to increase the safety of high-speed train bogie frames. In (Shahidi et al., 2015), was presented a datadriven method for bogie component fault classification based on mutual information (MI) measure feature selection and SVM. Features were extracted from acceleration signals and were divided into training and testing datasets. Features were ranked based on their similarity degree to the classified fault state by MI algorithm and were fed into SVM for bogie fault classification.

\subsection{Axles and Bearings}

One of the critical components of high-speed trains is rolling element bearings. In (Pennacchi et al., 2013), was studied rolling element bearings diagnostics of high-speed trains. They built experimental test-rig to collect vibration signals for bearing diagnostics. Different signal processing techniques were adopted to process vibration signals for fault detection. Spectral kurtosis algorithm was used to detect bearing faults. Furthermore, based on fault detection results they also analyzed sensitivity metrics of sensors to the bearing faults to reduce the number of sensors employed in diagnostics. Symonds et al. (2015) studied axle-bearing damage analysis. Using collected vibration signals, tested different techniques to identify bearing failure causes by identifying physical damage links between CM signals. Xie et al. (2015) presented health state diagnostics methodology for high-speed train axles. Temperature CM data collected from test line underwent the pre-processing step to replace zero values and noise cancellation by adopting interpolation and discrete wavelet transform techniques. Smoothed temperature data were used to calculate rising rates by a presented methodology 
for axle health state evaluation and it was concluded that this approach could be used effectively and was more practical when compared to other diagnostics approaches. In (Yi, Lin, Zhang, \& Ding, 2015) was presented a novel approach for fault diagnostics of railway axle bearings based on ensemble empirical mode decomposition (EEMD) and Hilbert transform (HT) signal processing techniques. To overcome the limitations of EEMD, which decomposes signals into varying intrinsic modes, they proposed an improved EEMD approach by introducing

\begin{tabular}{|c|c|c|}
\hline Component & Failure mode & Failure mechanisms \\
\hline \multirow[b]{2}{*}{ Bogie frame } & Surface defects & $\begin{array}{l}\text { - Fatigue cracks propagation } \\
\text { - Impacts }\end{array}$ \\
\hline & Loss of structural integrity & $\begin{array}{l}\text { - Fatigue cracks propagation } \\
\text { - Impacts } \\
\text { - Thermal shocks } \\
\text { - Material deformations (Yielding, creep) }\end{array}$ \\
\hline \multirow[t]{2}{*}{ Wheel } & Surface defects & $\begin{array}{l}\text { - Shelling } \\
\text { - Spalling } \\
\text { - Scaling } \\
\text { - Metal build-up } \\
\text { - Fatigue crack propagation }\end{array}$ \\
\hline & Subsurface defects & - Fatigue crack propagation \\
\hline \multirow[t]{2}{*}{ Axle bearing } & Bearing rotates with difficulty or cannot rotate & $\begin{array}{l}\text { - Fatigue (Subsurface initiated fatigue / Surface- } \\
\text { initiated fatigue) } \\
\text { - Wear (Abrasive wear / Adhesive wear) } \\
\text { - Fracture and Cracking (Forced fracture / Fatigue } \\
\text { fracture / Thermal cracking) }\end{array}$ \\
\hline & Bearing rotates with difficulty or cannot rotate & $\begin{array}{l}\text { - Fatigue (Subsurface initiated fatigue / Surface- } \\
\text { initiated fatigue) } \\
\text { - Wear (Abrasive wear / Adhesive wear) } \\
\text { - Fracture and Cracking (Forced fracture / Fatigue } \\
\text { fracture / Thermal cracking) }\end{array}$ \\
\hline Axle box & Surface defects & - Fatigue crack propagation \\
\hline Inner and outer springs & Reduction of the primary suspension effect & $\begin{array}{l}\text { - Fatigue crack propagation } \\
\text { - Material deformation (Yielding, creep) }\end{array}$ \\
\hline Centering springs & Reduction of the centering effect & $\begin{array}{l}\text { - Fatigue crack propagation } \\
\text { - Material deformation (Yielding, creep) }\end{array}$ \\
\hline Primary damper & Reduction of the damping effect & $\begin{array}{l}\text { - Seals wear } \\
\text { - Leakage }\end{array}$ \\
\hline Emergency springs & Reduction of emergency suspension effect & $\begin{array}{l}\text { - Fatigue crack propagation } \\
\text { - Material deformation (Yielding, creep) }\end{array}$ \\
\hline Air spring & Reduction of the secondary suspension effect & $\begin{array}{l}\text { - Leakage } \\
\text { - Fatigue crack propagation }\end{array}$ \\
\hline Vertical damper & Reduction of the vertical damping effect & $\begin{array}{l}\text { - Seals wear } \\
\text { - Leakage }\end{array}$ \\
\hline Lateral damper & Reduction of the lateral damping effect & $\begin{array}{l}\text { - Seals wear } \\
\text { - Leakage }\end{array}$ \\
\hline \multirow[t]{2}{*}{ Gearbox } & Gearbox rotates with difficulty or cannot rotate & $\begin{array}{l}\text { - Bearings: } \\
\text { O Fatigue (Subsurface initiated fatigue / Surface- } \\
\text { initiated fatigue) } \\
\text { ○ Wear (Abrasive wear / Adhesive wear) } \\
\text { Fracture and Cracking (Forced fracture / Fatigue } \\
\text { fracture / Thermal cracking) } \\
\text { - Gears: } \\
\text { O Subsurface initiated bending fatigue } \\
\text { O Scuffing } \\
\text { O Fretting corrosion } \\
\text { O Micropitting/Macropitting } \\
\end{array}$ \\
\hline & Bad or insufficient lubrication & $\begin{array}{l}\text { - Oil reservoir leakage } \\
\text { - Grease quality degradation } \\
\text { o High Temperatures Ageing } \\
\text { o Mechanical Ageing } \\
\text { o Contamination }\end{array}$ \\
\hline
\end{tabular}

Table 4. Bogie component failure modes and mechanisms. 
intrinsic mode function (IMF) confidence index to make EEMD automatic in IMF selection without any user assistance. And those selected IMFs were fed to HT to extract time-varying characteristics of signals for bearing fault diagnostics. Vibration signals were collected from test rig in different wheel speeds to analyze three type of artificially created bearing failure types under different operational modes. Improved EEMD with HT was effectively used for the axle bearing diagnostics. Zerbst et al. (2013) presented an overview paper on railway axles where they described different axle failure scenarios and impacts that can reduce fatigue strength during operation.

\subsection{Gearbox}

Due to the environment, high speed and complex structure, gearbox performance can be affected, threatening safety and reliability of high-speed trains. In (B. Zhang, Tan, \& Lin, 2016), was studied high-speed train gearbox crack fault detection. They analyzed vibration signals collected from gear box body and train speed under different speeds, they believed that cracks cause on gearbox body were highly correlated with wheel-rail contact. Vibration signals were analyzed in three conditions, different speed levels, different measurement points and in using different gear boxes. They used the low-pass filtering technique to extract frequency features from vibration signals for crack fault detection. And concluded the paper with three suggestions to resolve the gear box structure crack problem, which are; improvement on the gear box structure length, viewpoint shape modification and improvement of weak points on body structure. In (Henao, Kia, \& Capolino, 2011), was presented noninvasive measurement method in mechanical transmission of high-speed railway traction systems (RTS) for gear fault diagnostics. It was stated that gearbox torsional vibration had an influence on torque and it could be used for gear monitoring by demonstrating traction motor as a torque sensor based on electromagnetic-torque estimator without using any extra sensors. All measurements were carried out in reduced-scale of RTS in a laboratory environment, due to the high cost of performing different tests on real RTS. They also installed real sensors on different parts of gear box and torque to investigate correlations between estimated signals and real signals. Stationary component of estimated and measured torque vibration signals were analyzed by fast Fourier transform (FFT) and non-stationary frequencies were analyzed by spectrogram technique. All measurements were carried out to investigate effects of tooth damage and surface-wear faults on RTS gears and it was concluded that estimated electromagnetic torque and stator current signatures could be effectively used for gearbox fault diagnostics without installing physical sensors. In (Morgado, Branco, \& Infante, 2008), was studied driving gearbox body crack problems in locomotives. They collected strain measurements via rosettes installed on gear box body for fatigue stress analysis from two routine locomotive journeys. Measured strains from gear box body were used to analyze fatigue stress and linear Miner's rule was adopted for fatigue crack calculation. They also investigated critical areas on box body where possible fatigue crack might occur by performing stress analysis on gear box body.

\subsection{Wheels and Suspension}

Railway wheel maintenance is essential in terms of safety and reliability of trains. Cremona et al. (2016) proposed a novel approach in wheel wear RUL prediction based on local contact model and Archard's equation. The proposed methodology is integrated with $\mathrm{K}$ wear coefficient on wear prediction. The approach was validated by the real case study and prediction results were better than the methods proposed in the literature. Simple railway wheel diagnostics was presented in (Skarlatos, Karakasis, \& Trochidis, 2004) based on fuzzy-logic. Authors installed two accelerometers on the rail to track vibration signals of wheel-rail interaction. Vibration signals were collected from defected wheels and healthy wheels under different train speeds. Fuzzy-logic was used to detect the level of damage on the wheel to plan maintenance activities. In (Wei et al., 2012), was studied fiber Bragg grating sensors (FBG) ability to detect wheel defects based on real-time condition monitoring. Wu et al. (2015) studied incipient failure detection and estimation of a closed-loop secondary suspension system for high-speed trains. Authors, first of all, built a dynamic model of the suspension system and then proposed total measurable fault information residual (ToMFIR) estimation method. The suspension system was simulated by Matlab/Simulink with external disturbance parameters to generate faulty signals for their study. They claimed that proposed approach was able to detect and estimate propagating faults. Alfi et al. (2011) presented condition monitoring approach for bogie suspension component fault detection and isolation using model-free and model-based methods. The model-free approach is a data-driven method based on Random Decrement Technique (RDT) signal processing technique. Lateral acceleration measurement signals were processed to extract features for bogie incipient failure detection which was virtually measured by simulation. A model-based approach is a combination of EKF and Bayesian statistics. Physical suspension system model was built and simulated to generate different faulty virtual measurements. Both proposed approaches performed well in incipient fault detection. Fault detection and isolation study were also carried out in (Ding \& Mei, 2008)(Mei \& Ding, 2008). Authors carried out fault detection and isolation for dampers which are one of the components of primary suspension systems. Cross-correlation function was used to detect changes from the acceleration signals. Fault detection methodology was effectively proven on a simulated dataset. Melnik et al. (2014) developed an online monitoring system for suspension fault detection. Different acceleration signals were simulated by artificially creating faulty signals by playing with stiffness and damping parameters of the suspension system. For real dataset, they acquired acceleration signals for primary suspension via sensors located on the frame of bogie and for secondary suspension via sensors installed on the car body. Extracted features from both scenarios were used in fault detection by calculating Euclidean distances between 
faulty signal and a normal one for suspension diagnostics. Interacting multiple-model (IMM) approach was used to detect faults in lateral dampers of the secondary suspension system in (Tsunashima \& Mori, 2010). Measurement data were generated by full-vehicle model simulation for fault detection. IMM model was integrated with KF to update estimation model. And it was concluded that proposed approach could efficiently detect suspension system faults.

It is important to note that, prognostics research conducted for train components is rarely mentioned in the literature when compared with rotatory machinery applications. Some of the reasons could be due to difficulty in either building expensive test rigs or real-time monitoring, difficulty in data acquisition process and harsh operational conditions for prognostics of bogie components.

\section{PHM IMPLEMENTATION STEPS FOR INDUSTRY PRACTITIONERS}

Many important issues have been covered in the literature, such as prognostics approaches, data extraction techniques, prognostics methods pros and cons nearby PHM implementation that attempted to resolve challenges faced in engineering applications (Sikorska et al., 2011; Jardine et al., 2006; Lee et al., 2014). There are still important points to be clarified to make PHM more practical and easily understandable by industry. All systems either complex or simple, are non-linear which makes systemlevel health assessment applicability difficult. Due to this reason, we have proposed a simplified PHM design to guide industry to understand and apply PHM very effectively. The PHM implementation steps are as follows (see Fig. 18);
Critical component identification: to identify critical components which have high maintenance and failure consequences.

Sensor selection: to perform CM, we need robust and easily applicable sensors to get good quality data. Data preprocessing: to remove noise from raw data and perform data analysis for feature evaluation to get better prognostics feature.

Hybrid prognostics approach and tool evaluation: proposes a suitable model for bogie component RUL prediction and to select an appropriate tool based on evaluation combining practitioners' knowledge with tool performance matrices.

Maintenance decision making: to make better maintenance decision based on RUL information. This part is out of the scope of this review paper and is therefore not evaluated here.

\subsection{Component Criticality Analysis}

Component criticality analysis can be assumed as the starting point before PHM implementation. Industrial complex systems are composed of many assets that were designed to perform specific jobs during their useful life period. Each component has its own cost but some have a higher cost of system downtime when failed. The goal of critical asset identification process is to identify an asset which has a substantial impact on system functioning in terms of maintenance and failure consequences. Failure of such components may result even in catastrophic accidents. ISO standards factors (ISO 17359:2011, n.d.) for machine

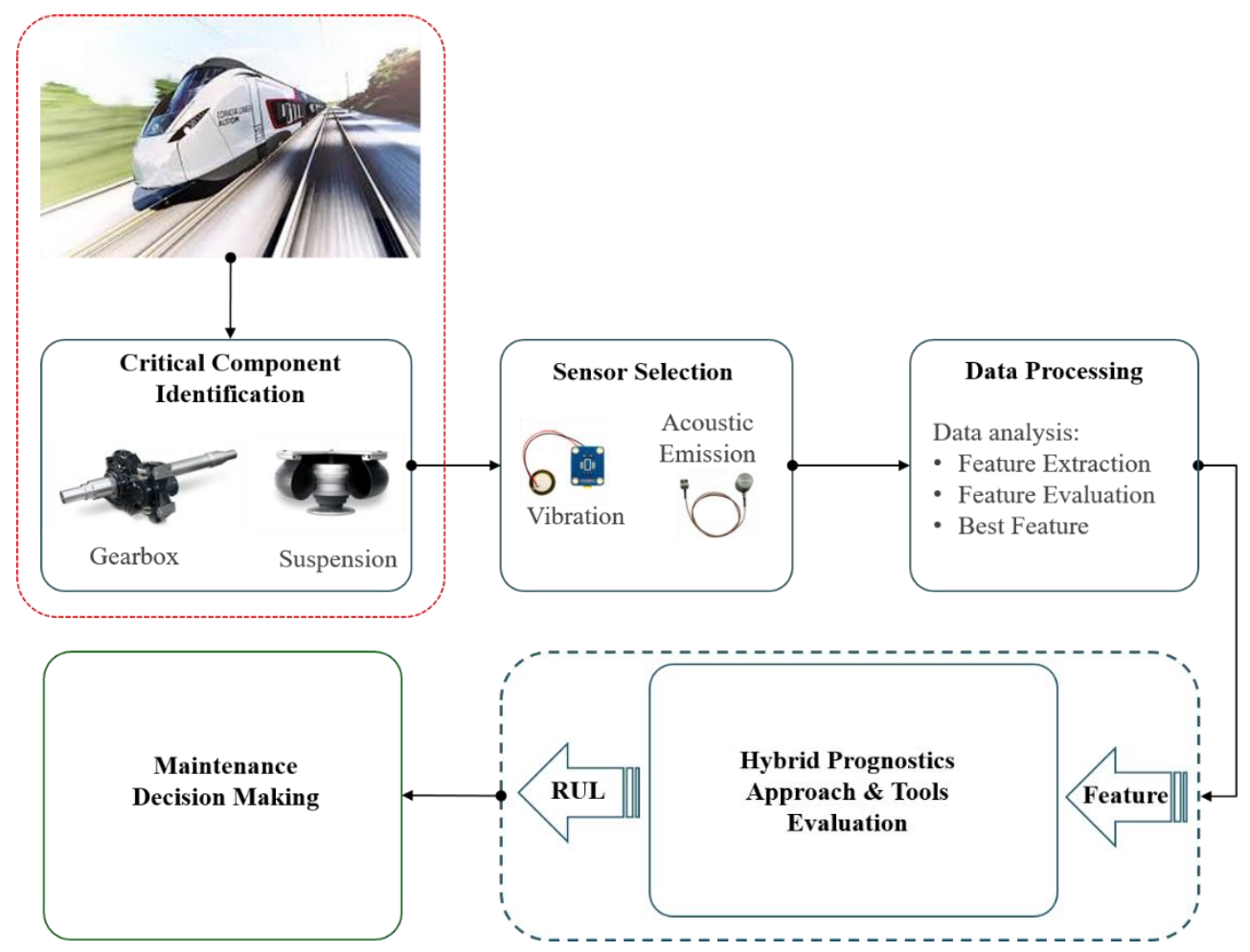

Fig. 18. PHM implementation steps. 
criticality assessment can be summarized as follows: machine downtime cost or lost production cost, machine failure rate and mean time spent to repair, machine replacement and maintenance cost, system monitoring cost and environmental impacts and machine safety. Using one or a combination of these factors, one can build a component priority list for machine health assessment. An example for critical components of high-speed train bogie is depicted in Fig. 19.

In (Lee et al., 2014), was presented a good example for component criticality analysis and identification for 890 SW Robots, which is illustrated in Fig. 20.They divided this graph into 4 quadrants, where each has its own meaning and suitable maintenance strategy for component falling into the associated quadrant. And each quadrant can be explained as follows:

Quadrant 1 has high fault frequency and high system downtime. Quadrant 2 has high fault frequency but low system downtime. Quadrant 3 has low fault frequency and low system downtime. Quadrant 4 has low fault frequency but high system downtime where more critical components fall in this area. As explained in this example, criticality of components is highly dependent on average downtime rather than fault frequency. It is important to note that critical component identification analysis is an important step for PHM, in terms of reliability, availability and safety issues for complex systems.

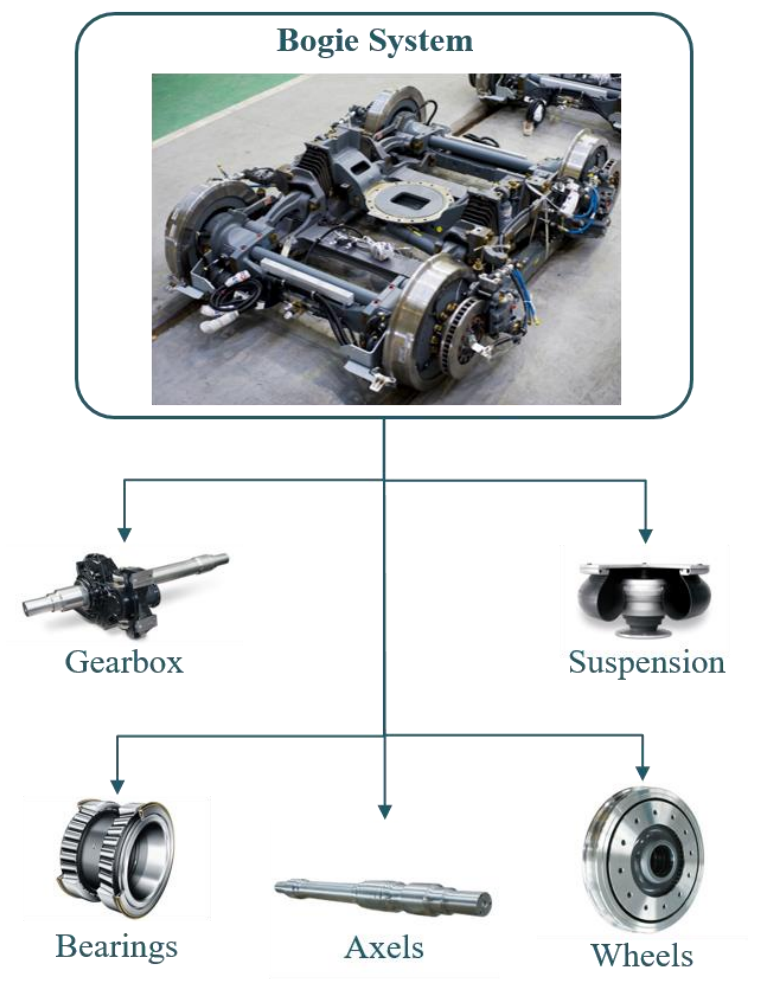

Fig. 19. Critical components for high-speed train bogie.

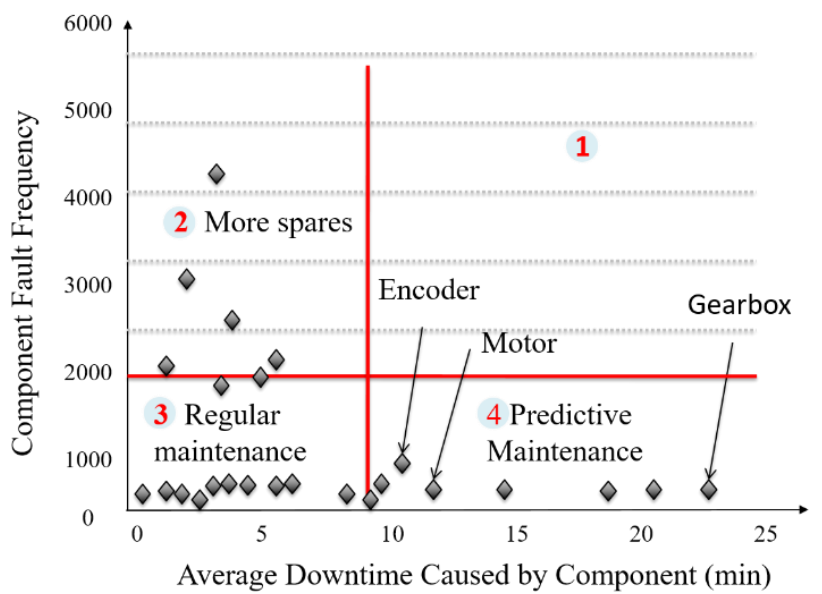

Fig. 20. Critical component identification based on 4 quadrants (modified from (J. Lee et al., 2014)).

\subsection{Right Sensor Selection for Condition Monitoring}

After identifying the critical component(s) before conducting PHM, one should deal with the sensor selection issue also. We all know that diagnostics and prognostics involve many complex tasks such as sensor selection, data acquisition, feature extraction, model selection and decision making. A performance metric of PHM approaches does not rely only on the diagnostics and prognostics tools used, but also on the performance metrics of sensors installed on the complex systems (see Fig. 21).

Camci et al. (2016) analyzed different prognostics methods for railway turnout systems by individually analyzing CM data collected by 5 different sensors installed on a point machine. First of all, they compared different sensors based on their cost, installation and usage simplicity. After data collection process, performed prognostics and analyzed prediction accuracy results. Each sensor was evaluated based on the RUL performance results and their ability to represent system degradation efficiently. Initial evaluation of sensors they presented is shown in Table 5 based on authors' findings.

Installation column metrics depends on the ability of the technician mounting time and assistance. If its mountable without any modification, then it is considered as 'easy'. If the installation is more than 2 hours and needs an extra labor, then it was considered as 'difficult'. Where sensitivity column indicates sensor output fluctuations or changes after artificially fault creation process. If the change in output signal varies between 3 and $10 \%$, then it is considered as 'moderate'. If output signal change is less than $5 \%$, then it is accepted as 'low'. If sensors do not reflect any change in an output signal, then it is accepted as 'limited'. For the detailed information, interested readers are referred to the original paper. (G. Zhang, 2005) presented a comprehensive research on sensor localization 


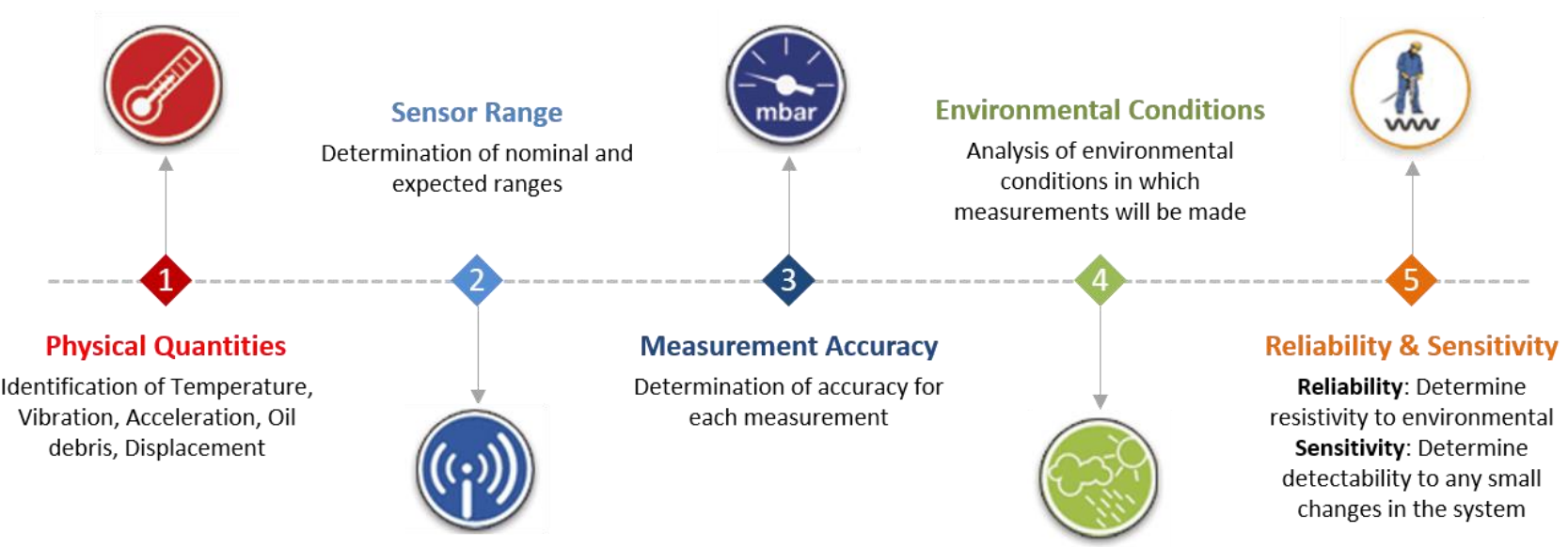

Fig. 21. Performance metrics for sensor selection.

\begin{tabular}{|c|c|c|c|c|}
\hline Sensor & Cost (euro) & $\begin{array}{l}\text { Installation } \\
\text { simplicity }\end{array}$ & Reliability & $\begin{array}{l}\text { Failure } \\
\text { sensitivity }\end{array}$ \\
\hline Force & $360+$ installation & Difficult & Reliable & High \\
\hline Current & 30 & Easy & Reliable & Low \\
\hline Voltage & 50 & Easy & Reliable & Low \\
\hline Proximity & 20 & Easy & $\begin{array}{l}\text { Reliable for short } \\
\text { distance }\end{array}$ & Limited \\
\hline Linear ruler & 250 & Moderate & Reliable & Moderate \\
\hline $\begin{array}{l}\text { Rotary encoder motor speed }+ \\
\text { position }\end{array}$ & $50+$ installation & Difficult & Reliable & Low \\
\hline $\begin{array}{l}\text { String encoder Gearbox output } \\
\text { position }+ \text { speed }\end{array}$ & $50+$ installation & Difficult & Reliable & Low \\
\hline
\end{tabular}

Table 5. Sensor comparison for prognostics ( adapted from (Camci et al., 2016)).

and selection problem for fault diagnostics purposes. Right sensor selection could be performed as in this example. There are many issues to be considered in right sensor selection depending on the application area, such as installation and maintenance cost, fault detectability and cost of redundant sensors in the case of sensor failures, etc. which need further investigation.

\subsection{Data Preprocessing}

Data preprocessing involves data cleaning for errors/noise cancellation and data analysis for deep interpretation of acquired signals. Detailed explanation for data preprocessing is presented in subsection 2.2. The feature selection methods are categorized as filter, wrapper and embedded methods (Chandrashekar \& Sahin, 2014) in the literature.

Filter feature selection methods use general properties of the variables to eliminate the least interesting ones depending on some ranking threshold (e.g. correlation, monotonicity and entropy etc.). In (H. Li et al., 2014) features were selected based on entropy information for chatter classification in milling machines by ANN whereas features which are too sensitive to the system health level were selected using Euclidean distance technique for failure diagnostics of gearboxes. Not all sensors monitoring the system provide useful information about system failure progression. A study on sensor selection methodology using entropy criteria was also presented for aircraft engine condition monitoring purpose (L. Liu et al., 2015). Atamuradov and Camci (2016), presented a novel feature evaluation approach considering features' dynamic behavior. Feature evaluation methodology is based on time series segmentation integrated with data fusion. It was evaluated using simulated and real Li-ion degradation data with accurate SoH estimation result. An online PCA was introduced to handle feature goodness in (Boutsidis \& Garber, 2015). Feature selection was performed based on predictability criteria for prognostics of bearings in (Javed et al., 2011).

Wrapper feature selection methods use searching algorithms (e.g. heuristic search algorithm) in variables' interaction evaluation to find the optimum number of variables that maximizes the objective function of the given algorithm. In (Boukra \& Lebaroud, 2014), a new feature selection methodology is presented adopting Particle Swarm Optimization (PSO) algorithm to figure out a good prognostics feature and employing particle filtering for remaining useful life prediction. Extracted features may have partial information about the system health state having low monotonicity property. Statistical degradation features from vibration, current and pressure measurements were extracted in (Liao, 2014) and a genetic algorithm was used to discover a new prognostics feature by data fusion. A fused feature having high monotonicity value was determined as a good prognostics feature. 
Embedded feature selection methods combine the advantages of previous two methods in feature selection. A novel feature selection methodology was proposed in (B. Li et al., 2011) based on combination of filter and wrapper methods to enhance the fault classification accuracy for gearbox diagnostics.

Performing diagnostics and prognostics analysis solely using one single feature may not be enough to characterize whole system degradation. Combining different sensory time series to achieve a more reliable prognostics feature is known as feature fusion. After extracting different nonstationary parameters from an aircraft engine, RUL prediction with Kalman Filter algorithm was performed using information fusion and superstatistics (Junqiang L. et al., 2014). Data-level based feature fusion was developed for prognostics of aircraft gas turbine (K. Liu, Gebraeel, \& Shi, 2013). Methane compressor prognostics was presented using multi-nonlinear regression model based on feature fusion by neural networks (Niu \& Yang, 2010). Different degradation features obtained from aged batteries under different operational modes were compared and $\mathrm{SoH}$ of aged batteries was estimated by data fusion in (Williard, He, Osterman, \& Pecht, 2013).

An accurate prediction of RUL depends on good prognostic features which emphasize the importance of feature evaluation for prognostics. Feature evaluation and selection for prognostics is a newly developing research area which needs further investigations and developments.

\subsection{Hybrid Prognostics Approach and Tool Evaluation for Bogie Monitoring}

One of the crucial steps of PHM is selecting the right prognostics model for component health assessment. In the literature, a lot of research has been conducted in a hybrid approach which is a combination of heterogeneous models that leverage merits of different approaches to making robust RUL predictions. Due to better performance of this model here we propose a hybrid prognostics approach which combines data-driven and physics-based prognostics models for bogie component health assessment. A general scheme of hybrid prognostics approach is depicted in Fig. 22.

To apply hybrid prognostics approach to bogie health monitoring one should follow the steps which were illustrated in Fig. 22, stated as : bogie critical components identification (gearbox, suspension, wheels, axles, and bearings), appropriate sensor selection (vibration and acoustic sensors), data analysis based on feature evaluation (monotonicity, prognosability, and trendability) to select best prognostics feature and select right prognostics tool for RUL prediction. The main goal of PHM technology is to support maintenance practitioners to make right and fast decisions. To make right and fast decisions depend on the accurate results of component RULs. To make accurate component RUL prediction we need to select suitable prognostics tools which have accurate estimations about future failure progression of the system or component. Prognostics tool evaluation and selection are performed based on characteristics of data, expert-knowledge, model structure and associated tool performance metrics. Evaluation and selection process mostly rely on expertknowledge and experience. To perform tool evaluation and selection to suggest suitable prognostics tool, it is necessary to form a general ranking system that combines user requirements and tool performance matrices. A paper on tool performance metric evaluation for data-driven prognostics tools can be found in (Saxena et al., 2009).

In this paper we propose a new prognostics tool evaluation matrix enabling user to select appropriate tool for their prognostics tasks which involve basic requirements and tool efficiency parameters. Since model-based and datadriven prognostics tools have different performance criteria and expert-knowledge requirements, they were evaluated in different matrices separately.

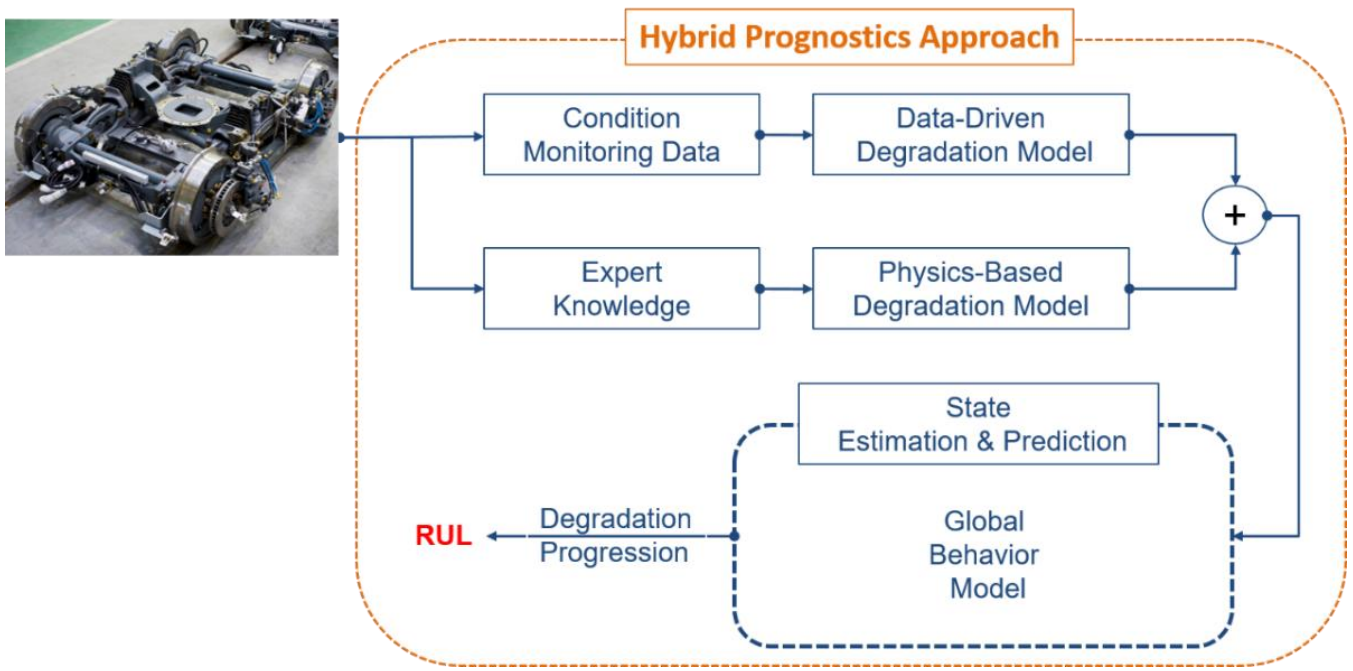

Fig. 22. Hybrid prognostics approach for bogie monitoring. 


\subsubsection{Model-based Prognostics Tool Evaluation}

Basic requirements. In this section we guide an engineer and/or practitioner in industry to have some prior information before tool selection. Basic requirement criteria parameters are as follows:

Expert-knowledge - states prior knowledge that practitioner and/or maintenance engineer has to have, such as mathematics, differential equations, statistics and probability theory before model development.

Model structure - is information required about the model structure to use expert-knowledge in modeling physical phenomena.

Data - defines characteristics of data that is collected from the system under inspection, stating its noise level parameter whether it is Gaussian or non-Gaussian. Because not all model-based tools are able to deal with the same type of noise and system behavior.

Tool efficiency. This section evaluates tools based on their performance and efficiency metrics. An appropriate weight is assigned to the corresponding metrics in the given

Run time - it describes the running time of prognostics tool in estimation and prediction process. Run time metric is evaluated by assigning associated weights based on their speed in completing assigned task. If one of the modelbased prognostics tools need more time to perform estimation and/or prediction, then it's accepted as a disadvantage for the corresponding tool and lesser weight is assigned. Weight assignment is done based on those criteria: if running time is high (red colored inFig. 23) then we assign numerical value 1 , if it is medium then 2 , if it has lower (blue colored in Fig. 23) running time which predicts in shorter time, then we assign numerical value 3 as a weight parameter.

Accuracy - describes the estimation and/or prediction accuracy of tools in prognostics tasks. If estimation and/or prediction accuracy are high, then it should have a maximum weight for evaluation.

Robustness - describes the ability of prognostics tool in dealing with the noise level and system uncertainty situations. If prognostics tool is robust in noisy data, then it should have maximum weight parameter.

Prediction horizon - describes prognostics tool ability to predict future failure evolution of assets under the investigation that is compatible with a time scale of asset residual life. If the prediction horizon is long, then that prognostics tool should be ranked with higher weights.

Tool ranking - after considering tool efficiency or performance metrics we can rank the corresponding tool by summing up assigned weights. In this section, the user can choose an appropriate tool with higher ranking value for their application. One can choose a prognostics tool with the higher ranking value from the matrix given in Fig. 23 below. Based on the weights assigned, tools which have higher ranking values are Paris' law, Forman law, fatigue spall propagation and $\mathrm{PF}$. If the user requirements meet those criteria, then one of those prognostics tools can be selected and applied in prognostics task.

\subsubsection{Data-driven Prognostics Tool Evaluation}

Data-driven prognostics tool evaluation matrix has the same parameters as was explained in section 8.4.1. Only the difference from model-based prognostics tool evaluation is the learning time metric under tool efficiency column.

Learning time - describes the time for data-driven prognostics to train the measurement model using CM data. Learning time depends on the size, type, and quality of $\mathrm{CM}$ data. If the time spent on learning or training the tool is lower (blue colored in Fig. 24), then it should have higher weights in ranking. Based on the weights assigned, tools which have higher ranking values for data-driven tools are Gaussian Mixture, Gaussian Process Regression, ANN,

\begin{tabular}{|c|c|c|c|c|c|c|c|c|c|}
\hline \multirow{3}{*}{ Tools } & \multicolumn{4}{|c|}{ Basic Requirements } & \multicolumn{4}{|c|}{ Tool Efficiency } & \multirow{3}{*}{$\begin{array}{c}\text { Tool } \\
\text { Ranking }\end{array}$} \\
\hline & \multirow{2}{*}{$\begin{array}{c}\text { Expert } \\
\text { Knowledge }\end{array}$} & \multirow{2}{*}{$\begin{array}{c}\text { Model } \\
\text { Structure }\end{array}$} & \multicolumn{2}{|c|}{ Data } & \multirow{2}{*}{$\begin{array}{l}\text { Run } \\
\text { Time }\end{array}$} & \multirow{2}{*}{ Accuracy } & \multirow{2}{*}{ Robustness } & \multirow{2}{*}{$\begin{array}{c}\text { Prediction } \\
\text { Horizon }\end{array}$} & \\
\hline & & & Gaussian & Non Gaussian & & & & & \\
\hline Paris' Law & $\checkmark$ & $v$ & 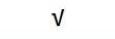 & $\mathrm{x}$ & High & High & High & Long & 10 \\
\hline Forman Law & $v$ & $v$ & v & $\mathrm{x}$ & High & High & High & Long & 10 \\
\hline $\begin{array}{l}\text { Fatigue Spall Initiation } \\
\text { /Progression }\end{array}$ & $v$ & $v$ & $v$ & $\mathrm{x}$ & High & High & High & Long & 10 \\
\hline Kalman Filter & $v$ & $v$ & $v$ & $x$ & Medium & Medium & Medium & Short & 7 \\
\hline Particle Filter & $v$ & $v$ & $x$ & $v$ & High & High & High & Long & 10 \\
\hline
\end{tabular}

\begin{tabular}{|c|c|c|c|c|}
\hline \multicolumn{2}{|c|}{ High } & \multirow{2}{*}{ Medium } & \multicolumn{2}{|c|}{ Low } \\
\cline { 5 - 6 } & & & & \\
\hline 1 & 3 & 2 & 1 & 3 \\
\hline
\end{tabular}

\begin{tabular}{|c|c|c|}
\hline Long & Medium & Short \\
\hline 3 & 2 & 1 \\
\hline
\end{tabular}

Fig. 23. Model-based prognostics tool evaluation and selection matrix. 


\begin{tabular}{|c|c|c|c|c|c|c|c|c|c|c|c|c|c|c|c|}
\hline & & & \multicolumn{7}{|c|}{ Basic Requirements } & \multicolumn{5}{|c|}{ Tool Efficiency } & \multirow{3}{*}{$\begin{array}{c}\text { Tool } \\
\text { Ranking }\end{array}$} \\
\hline \multirow{2}{*}{\multicolumn{3}{|c|}{ Tools }} & \multirow{2}{*}{\multicolumn{2}{|c|}{$\begin{array}{c}\text { Expert } \\
\text { Knowledge }\end{array}$}} & \multirow{2}{*}{\multicolumn{2}{|c|}{$\begin{array}{c}\text { Model } \\
\text { Structure }\end{array}$}} & \multicolumn{3}{|c|}{ Data } & \multirow{2}{*}{$\begin{array}{l}\text { Run } \\
\text { Time }\end{array}$} & \multirow{2}{*}{$\begin{array}{l}\text { Learning } \\
\text { Time }\end{array}$} & \multirow[b]{2}{*}{ Accuracy } & \multirow[b]{2}{*}{ Robustness } & \multirow{2}{*}{$\begin{array}{l}\text { Prediction } \\
\text { Horizon }\end{array}$} & \\
\hline & & & & & & & Linear & & $\begin{array}{l}\text { Non- } \\
\text { Linear }\end{array}$ & & & & & & \\
\hline \multicolumn{3}{|c|}{ ARMA } & & $x$ & \multicolumn{2}{|l|}{$x$} & $\checkmark$ & & $x$ & Low & Low & Medium & Low & Short & 10 \\
\hline \multicolumn{3}{|c|}{ Match Matrix } & & $x$ & \multicolumn{2}{|l|}{$x$} & $\mathrm{x}$ & & $v$ & Low & Low & High & Medium & Medium & 13 \\
\hline \multicolumn{3}{|c|}{ T. Series Analysis } & & $\mathrm{x}$ & \multicolumn{2}{|l|}{$\mathrm{x}$} & $v$ & & $\mathrm{x}$ & Low & Low & Medium & Low & Short & 10 \\
\hline \multicolumn{3}{|c|}{ Logistic Reg. } & & $\mathrm{x}$ & \multicolumn{2}{|l|}{$\mathrm{x}$} & $v$ & & $x$ & Low & Low & High & Low & Short & 11 \\
\hline \multicolumn{3}{|c|}{ Gaussian M. } & & v & \multicolumn{2}{|l|}{$\checkmark$} & $x$ & & $v$ & Low & High & High & High & Medium & 15 \\
\hline \multicolumn{3}{|c|}{ Gaussian P.R/P. } & & $\checkmark$ & \multicolumn{2}{|l|}{$v$} & $x$ & & $v$ & Low & High & High & High & Medium & 15 \\
\hline \multicolumn{3}{|c|}{ ANN } & & $x$ & $v$ & & $\mathrm{x}$ & & $v$ & Medium & High & High & High & Long & 14 \\
\hline Fuz & zy L. & & & $\checkmark$ & v & & $\mathrm{x}$ & & v & High & Medium & High & High & Medium & 11 \\
\hline so & & & & $x$ & $x$ & & $\mathrm{x}$ & & $v$ & Low & Medium & High & Medium & Medium & 12 \\
\hline Bay & esia & & & $v$ & $v$ & & $\mathrm{x}$ & & $v$ & High & High & High & Medium & Long & 12 \\
\hline SVI & & & & $v$ & v & & $v$ & & $x$ & Low & High & High & Medium & Long & 14 \\
\hline $\mathrm{HN}$ & & & & $v$ & v & & $x$ & & $v$ & High & High & High & High & Long & 13 \\
\hline $\mathrm{Hi}$ & gh & & & w & Long & & edium & Short & & & & & & & \\
\hline & & & & & 3 & & 2 & 1 & & & & & & & \\
\hline 1 & 3 & 2 & 1 & 3 & & & & & & & & & & & \\
\hline
\end{tabular}

Fig. 24. Data-driven prognostics tool evaluation and selection matrix.

and SVM which is illustrated in Fig. 24. If the user requirements meet those criteria, then one of those prognostics tools can be chosen and applied in prognostics task.

\section{CONCLUSION}

PHM technology is employed widely to enhance system availability and safety and to analyze the system performance based on time series data acquired from different sensors depending on component functionality. We have presented a general view of PHM and its steps to provide prior knowledge for users, reviewed different PHM approaches under model-based, data-driven and hybrid models, and discussed their merits and drawbacks. We have also reviewed previous and on-going research in bogie components PHM to highlight problems faced in the railway industry. As a result of PHM literature review on bogie components, we noticed that nearly all research conducted in bogie health assessment is mostly limited to diagnostics rather than prognostics tasks. Since railway vehicle bogies are critical components, research on prognostics for asset health management is also crucial to provide a safe and comfortable ride for customers. Consequently, if somebody wants to implement PHM technology at system or component level, the first step is to identify critical components in the system that have a great impact on system functionality when they fail. The secondly step is to select and install right and robust sensors on the system for accurate CM. Collected CM data should be properly processed for good prognostics feature extraction, as a third step. Finally, a suitable PHM approach should be carefully selected based on the user requirements as well as a prognostics tools selection process. Since accurate prognostics results are based on the tools used in prediction, the tools evaluation and selection process is an important task in PHM implementation. Two separate prognostics tools evaluation matrices were presented, for both model-based and data-driven approaches. Prognostics tools were evaluated and ranked based on a combination of user knowledge and tool performance metrics. As a result of our investigation, we can conclude that further investigations and improvements should be carried out in following areas for bogie prognostics;

- High-speed train bogies have dynamic loads and environmental conditions which might affect component failures differently. To detect and monitor component behaviors under different loads, intelligent component criticality identification systems should be developed.

- Intelligent on-board or off-board data preprocessing systems which analyze CM data efficiently and extract better health indicators for prognostics should be designed.

Efficient and intelligent prognostics tool evaluation and selection systems that integrate user requirements with tool performance metrics, tool applicability, CM data characteristics and suggest a suitable tool for better component RUL prediction with minimum uncertainties should be developed.

\section{ACKNOWLEDGEMENTS}

This research was supported by a grant ENIT, Production Engineering Laboratory (LGP), funded by ALSTOM. 


\section{BIOGRAPHIES}

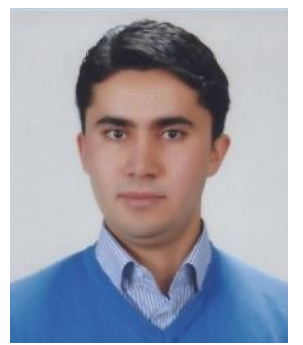

Vepa Atamuradov was born in Turkmenistan in 1986. He received his Ph.D. in Electrical-Computer Engineering from Selcuk University (2016), MS in Computer Engineering from Fatih University (2009) in Turkey, and BS in Computer Engineering from International Black Sea University in Tbilisi, Georgia (2007). He actively worked as an RA in both "Development of Failure Prognostics and Maintenance Planning System for Point Mechanisms in Railway (2008-2009)" and "Development of Design based State-of-Health and Remaining Useful Life Estimation Techniques for Battery Management Systems and Its Application to Rechargeable Batteries (2014-2016)" projects, granted by The Scientific and Technological Research Council (TUBITAK) of Turkey. Currently, he is working as a Postdoc research fellow on PHM of high-speed train bogies at Tarbes National School of Engineering (ENIT), France, since July 2016. His research interests include failure diagnostics and prognostics of Industrial systems using Machine Learning and Statistical Methods.

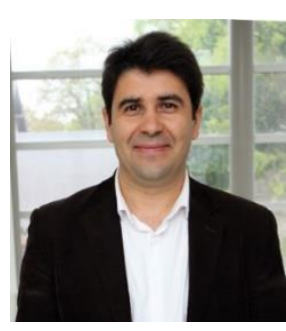

Kamal Medjaher is Professor at Tarbes National School of Engineering (ENIT), France, since February 2016. He conducts his research activities within the Production Engineering Laboratory (LGP). Before this position, he was Associate Professor at the National Institute of Mechanics and Microtechnologies in Besançon, France, from September 2006 to January 2016. After receiving an engineering degree in electronics, he received his MS in control and industrial computing in 2002 at "Ecole Centrale de Lille" and his Ph.D. in 2005 in the same field from University of Lille 1. Since September 2006, Kamal Medjaher leads research works in the field of Prognostics and Health Management of industrial systems.

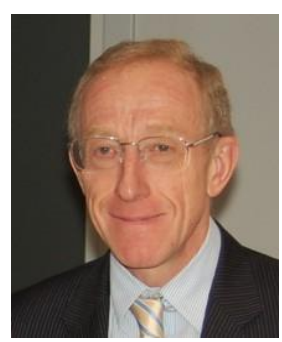

Pierre Dersin received his Ph.D. in Electrical Engineering from MIT (1980), MS in O.R. also from MIT. Engineer and Math BS from Brussels University, Belgium. Since 1990 with ALSTOM Transport, he has occupied several positions in RAMS, Maintenance and R\&D. Currently RAM (Reliability-AvailabilityMaintainability) Director and PHM (Prognostics \& Health Management) Director of ALSTOM Digital Mobility (the part of ALSTOM which acts as a catalyst for the 'digital revolution'). Also, Leader of ALSTOM 's Reliability \&
Availability Core Competence Network. Since April 2014, co-Director of the joint ALSTOM-INRIA Research Laboratory for digital technologies applied to mobility and energy. Prior to joining ALSTOM, he had worked on fault diagnostic systems for factory automation (with Engineering firm Fabricom), and earlier, at MIT LIDS, studied the reliability of electric power grids, as part of the Large Scale System Effectiveness Analysis Program funded by the US Department of Energy. His current research interests include the links between reliability engineering and PHM and the application of data science, machine learning and statistics to both fields, as well as systems optimization and simulation, including systems of systems. He is a member of the IEEE Reliability Society's Administrative Committee and IEEE Future Directions Committee and, as of January 2017, will be the IEEE Reliability Society Vice-President for Technical Activities. He was a Keynote speaker at the $2^{\text {nd }}$ European PHM Conference, Nantes, July 2014.

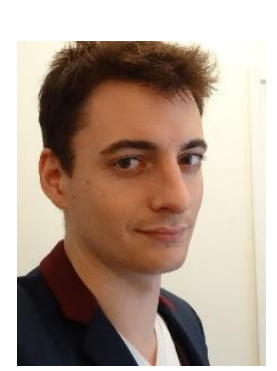

Benjamin Lamoureux was born in France in 1986. He received both a Master's Degree in Mechatronics from the University Pierre \& Marie Curie and a Master's Degree in Engineering from Arts \& Métiers ParisTech in Paris in 2010. From 2011 to 2014, he performed an industrial Ph.D. work in collaboration between Arts \& Métiers ParisTech and SAFRAN Aircraft Engines Villaroche (formerly SAFRAN Snecma). He received his Ph.D. in June 2014. In October 2014, he joined the PHM department of Alstom Saint-Ouen to extend his $\mathrm{Ph} . \mathrm{D}$. research in the railway domain, and he still occupies the same position today. His current research interests are machine learning, statistics, data science and computer science applied to PHM.

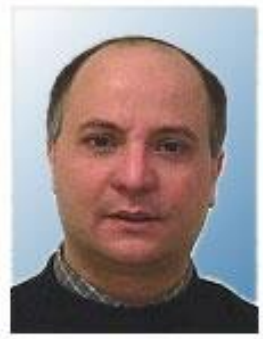

Noureddine Zerhouni received the Engineering degree from the National Engineers and Technicians Institute of Algiers, Algiers, Algeria, in 1985, and the Ph.D. degree in automatic control from the Grenoble National Polytechnic Institute, Grenoble, France, in 1991. He joined the National Engineering Institute of Belfort, Belfort, France, as an Associate Professor in 1991. Since 1999, he has been a Full Professor with the National Institute in Mechanics and Microtechnologies, Besançon, France. He is a member of the Department of Automatic Control and MicroMechatronic Systems at the FEMTO-ST Institute, Besançon. He has been involved in various European and national projects on intelligent maintenance systems. His current research interests include intelligent maintenance, and prognostics and health management. 


\section{REFERENCES}

Alfi, S., Bionda, S., Bruni, S., \& Gasparetto, L. (2011). Condition monitoring of suspension components in railway bogies. 5th IET Conference on Railway Condition Monitoring and Non-Destructive Testing (RCM 2011), 1-6.

An, D., Choi, J.-H., \& Kim, N. H. (2013). Prognostics 101: A tutorial for particle filter-based prognostics algorithm using Matlab. Reliability Engineering \& System Safety, 115, 161-169.

An, D., Kim, N. H., \& Choi, J. H. (2015). Practical options for selecting data-driven or physics-based prognostics algorithms with reviews. Reliability Engineering and System Safety, 133, 223-236.

Andre, D., Appel, C., Soczka-Guth, T., \& Sauer, D. U. (2013). Advanced mathematical methods of SOC and $\mathrm{SOH}$ estimation for lithium-ion batteries. Journal of Power Sources.

Atamuradov, V., \& Camci, F. (2016). Evaluation of Features with Changing Effectiveness for Prognostics. Annual Conference of the Prognostics and Health Management Society 2016.

Baraldi, P., Compare, M., Sauco, S., \& Zio, E. (2013). Ensemble neural network-based particle filtering for prognostics. Mechanical Systems and Signal Processing, 41(1-2), 288-300.

Baraldi, P., Mangili, F., \& Zio, E. (2015). A prognostics approach to nuclear component degradation modeling based on Gaussian Process Regression. Progress in Nuclear Energy, 78, 141-154.

Boukra, T., \& Lebaroud, A. (2014). Identifying New Prognostic Features for Remaining Useful Life Prediction. Power Electronics and Motion Control Conference and Exposition (PEMC), 2014 16th International, 1216-1221.

Boutsidis, C., \& Garber, D. (2015). Online Principal Component Analysis. Proceedings of the TwentySixth Annual ACM-SIAM Symposium on Discrete Algorithms., 887-901.

Bressel, M., Hilairet, M., Hissel, D., \& Ould Bouamama, B. (2016). Extended Kalman Filter for prognostic of Proton Exchange Membrane Fuel Cell. Applied Energy, 164, 220-227.

Burgess, W. L. (2009). Valve Regulated Lead Acid battery float service life estimation using a Kalman filter. Journal of Power Sources, 191(1), 16-21.

Camci, F., Eker, O. F., Baskan, S., \& Konur, S. (2016). Comparison of sensors and methodologies for effective prognostics on railway turnout systems. Proceedings of the Institution of Mechanical Engineers, Part F: Journal of Rail and Rapid Transit.
Campbell, G. S., \& Lahey, R. (1984). A survey of serious aircraft accidents involving fatigue fracture. International Journal of Fatigue, 6(1), 25-30.

Chandrashekar, G., \& Sahin, F. (2014). A survey on feature selection methods. Computers and Electrical Engineering, 40(1), 16-28.

Choi, Y., \& Liu, C. R. (2007). Spall progression life model for rolling contact verified by finish hard machined surfaces. Wear, 262(1-2), 24-35.

Coble, J. B., Ramuhalli, P., Bond, L. J., Hines, W., \& Upadhyaya, B. (2015). A review of prognostics and health management applications in nuclear power plants. International Journal of Prognostics and Health Management, 6(SP3), 1-22.

Coble, J., \& Hines, J. W. (2009). Identifying optimal prognostic parameters from data: a genetic algorithms approach. Proceedings of the Annual Conference of the Prognostics and Health Management Society, 1-11.

Cremona, M. A., Liu, B., Hu, Y., Bruni, S., \& Lewis, R. (2016). Predicting railway wheel wear under uncertainty of wear coefficient, using universal kriging. Reliability Engineering and System Safety, 154, 49-59.

Daigle, M., Sankararaman, S., \& Roychoudhury, I. (2016) System-level Prognostics for the National Airspace. Annual Conference of the Prognostics and Health Management Society 2016, 1-9.

Ding, X. J., \& Mei, T. X. (2008). Fault Detection for Vehicle Suspensions Based on System Dynamic Interactions. Procedings of the UKACC International Conference on Control. Retrieved from

Dong, H., Jin, X., Lou, Y., \& Wang, C. (2014). Lithiumion Battery State of health monitoring and Remaining Useful Life prediction based on Support Vector Regression-Particle Filter. Journal of Power Sources, 271, 114-123.

Dong, M., \& He, D. (2007). A segmental hidden semiMarkov model (HSMM)-based diagnostics and prognostics framework and methodology. Mechanical Systems and Signal Processing, 21(5), $2248-2266$.

Duong, P. L. T., \& Raghavan, N. (2017). Uncertainty quantification in prognostics: A data driven polynomial chaos approach. 2017 IEEE International Conference on Prognostics and Health Management, ICPHM 2017, 135-142.

Eker, O. F., Camci, F., Guclu, A., Yilboga, H., Sevkli, M., \& Baskan, S. (2011). A simple state-based prognostic model for railway turnout systems. IEEE Transactions on Industrial Electronics, 58(5), 17181726. 
Eker, O. F., Camci, F., \& Jennions, I. K. (2015). Physicsbased prognostic modelling of filter clogging phenomena. Mechanical Systems and Signal Processing, 75, 395-412.

Ferri, F. A. S., Rodrigues, L. R., Gomes, J. P. P., de Medeiros, I. P., Galvao, R. K. H., \& Nascimento, C. L. (2013). Combining PHM information and system architecture to support aircraft maintenance planning. 2013 IEEE International Systems Conference (SysCon), 60-65.

Forman, R. G. (1972). Study of fatigue crack initiation from flaws using fracture mechanics theory. Engineering Fracture Mechanics, 4(2), 333-345.

Gebraeel, N., Lawley, M., Liu, R., \& Parmeshwaran, V. (2004). Residual life predictions from vibrationbased degradation signals: A neural network approach. IEEE Transactions on Industrial Electronics, 51(3), 694-700.

Goebel, K., Eklund, N., \& Bonanni, P. (2006). Fusing competing prediction algorithms for prognostics. 2006 IEEE Aerospace Conference.

Gu, J., Vichare, N., Ayyub, B., \& Pecht, M. (2010). Application of Grey Prediction Model for Failure Prognostics of Electronics. International Journal of Performability Engineering, 6(5), 435-442.

Guan, X., Giffin, A., Jha, R., \& Liu, Y. (2012). Maximum relative entropy-based probabilistic inference in fatigue crack damage prognostics. Probabilistic Engineering Mechanics, 29, 157-166.

Guillén, A. J., Gómez, J. F., Crespo, A., Guerrerro, A., Sola, A., \& Barbera, L. (2013). Advances in PHM application frameworks: Processing methods, prognosis models, decision making. Chemical Engineering Transactions, 33, 391-396.

Halis Yilboga, Ömer Faruk Eker, A. G. F. C. (2010). Failure Prediction on Railway Turnouts Using TDNN_. 2010 IEEE International Conference on Computational Intelligence for Measurement Systems and Applications.

He, J., Lu, Z., \& Liu, Y. (2012). New Method for Concurrent Dynamic Analysis and Fatigue Damage Prognosis of Bridges. Journal of Bridge Engineering, 17(3), 396-408.

He, W., Williard, N., Osterman, M., \& Pecht, M. (2011). Prognostics of lithium-ion batteries based on Dempster-Shafer theory and the Bayesian Monte Carlo method. Journal of Power Sources, 196(23), 10314-10321.

Henao, H., Kia, S. H., \& Capolino, G. A. (2011). Torsionalvibration assessment and gear-fault diagnosis in railway traction system. IEEE Transactions on Industrial Electronics, 58(5), 1707-1717.
Hendricks, C., Williard, N., Mathew, S., \& Pecht, M. (2015). A failure modes, mechanisms, and effects analysis (FMMEA) of lithium-ion batteries. Journal of Power Sources, 297, 113-120.

Heng, A., Zhang, S., Tan, A. C. C., \& Mathew, J. (2009) Rotating machinery prognostics: State of the art, challenges and opportunities. Mechanical Systems and Signal Processing, 23(3), 724-739.

Hong-feng, W. (2012). Prognostics and Health Management for Complex system Based on Fusion of Model-based approach and Data-driven approach. Physics Procedia, 24, 828-831.

Hong, M., Wang, Q., Su, Z., \& Cheng, L. (2014). In situ health monitoring for bogie systems of CRH380 train on Beijing-Shanghai high-speed railway. Mechanical Systems and Signal Processing, 45(2), 378-395.

Hu, C., Youn, B. D., Wang, P., \& Taek Yoon, J. (2012). Ensemble of data-driven prognostic algorithms for robust prediction of remaining useful life. Reliability Engineering and System Safety, 103, 120-135.

Huang, T., Wang, L., Jiang, T., \& District, H. (2010). Prognostics of Products Using Time Series Analysis Based on Degradation Data. 2010 Prognostics and System Health Management Conference, Macao.

Irwin, G. R., \& Paris, P. C. (1971). CHAPTER 1 FUNDAMENTAL ASPECTS OF CRACK GROWTH AND FRACTURE. In Engineering Fundamentals and Environmental Effects (pp. 1-46).

ISO 13372:2012. (n.d.). Condition monitoring and diagnostics of machines-Vocabulary. Retrieved from http://viewer.afnor.org/Pdf/Viewer/?token=VR4Vvn prAPA1

ISO 13374-1:2003. (n.d.). Condition monitoring and diagnostics of machines - Data processing, communication and presentation - Part 1: General guidelines. $\quad$ Retrieved from http://viewer.afnor.org/Pdf/Viewer/?token=CBE$\mathrm{mBI} 2 \mathrm{~g} 7 \mathrm{w} 1$

ISO 13381-1:2005. (n.d.). Condition monitoring and diagnostics of machines - Prognostics - Part 1: General guidelines. Retrieved from http://viewer.afnor.org/Pdf/Viewer/?token=ou_CxV 4KW2s 1

ISO 17359:2011. (n.d.). Condition monitoring and diagnostics of machines- General guidelines. Retrieved from http://viewer.afnor.org/Pdf/Viewer/?token=35DEU CJIWQI1

J.Z. Sikorska , M. Hodkiewiczb, L. M. (2011). Prognostic modelling options for remaining useful life estimation.pdf. Mechanical Systems and Signal Processing 25 (2011) 1803-1836 Contents. 
Jardine, A. K. S., Lin, D., \& Banjevic, D. (2006). A review on machinery diagnostics and prognostics implementing condition-based maintenance. Mechanical Systems and Signal Processing, 20(7), 1483-1510.

Javed, K., Gouriveau, R., Zemouri, R., \& Zerhouni, N. (2011). Improving data-driven prognostics by assessing predictability of features. Annual Conference of the Prognostics and Health Management Society, 1-6.

Jouin, M., Gouriveau, R., Hissel, D., Péra, M. C., \& Zerhouni, N. (2014). Prognostics of PEM fuel cell in a particle filtering framework. International Journal of Hydrogen Energy, 39(1), 481-494.

Kalman, R. E. (1960). A New Approach to Linear Filtering and Prediction Problems. Journal of Basic Engineering, 82(1), 35.

Kan, M. S., Tan, A. C. C., \& Mathew, J. (2015). A review on prognostic techniques for non-stationary and nonlinear rotating systems. Mechanical Systems and Signal Processing, 62, 1-20.

Kandukuri, S. T., Klausen, A., Karimi, H. R., \& Robbersmyr, K. G. (2016). A review of diagnostics and prognostics of low-speed machinery towards wind turbine farm-level health management. Renewable and Sustainable Energy Reviews, 53, 697-708.

Khorasgani, H., Biswas, G., \& Sankararaman, S. (2016). Methodologies for system-level remaining useful life prediction. Reliability Engineering and System Safety, 154, 8-18.

Kimotho, J. K., \& Sextro, W. (2014). An approach for feature extraction and selection from non-trending data for machinery prognosis. Proceedings of the Second European Conference of the Prognostics and Health Management Society, 1-8.

Kumar, S., Torres, M., Chan, Y. C., \& Pecht, M. (2008). A hybrid prognostics methodology for electronic products. Proceedings of the International Joint Conference on Neural Networks, 3479-3485.

Lee, J., Wu, F., Zhao, W., Ghaffari, M., Liao, L., \& Siegel, D. (2014). Prognostics and health management design for rotary machinery systems - Reviews, methodology and applications. Mechanical Systems and Signal Processing, 42(1-2), 314-334.

Lee, S., Cui, H., Rezvanizaniani, M., \& Ni, J. (2012). Battery Prognostics: Soc and Soh Prediction. Proceedings of the ASME 2012 International Manufacturing Science and Engineering Conference, 1-7.

Li, B., Zhang, P. L., Tian, H., Mi, S. S., Liu, D. S., \& Ren, G. Q. (2011). A new feature extraction and selection scheme for hybrid fault diagnosis of gearbox. Expert
Systems with Applications, 38(8), 10000-10009.

Li, C. J., \& Lee, H. (2005). Gear fatigue crack prognosis using embedded model, gear dynamic model and fracture mechanics. Mechanical Systems and Signal Processing, 19(4), 836-846.

Li, H., Zhao, J., Yang, R., Zhao, J., \& Teng, H. (2014). Research on planetary gearboxes feature selection and fault diagnosis based on EDT and FDA. Proceedings of 2014 Prognostics and System Health Management Conference, PHM 2014, 178-181.

LI, Y., KURFESS, T. R., \& LIANG, S. Y. (2000) Stochastic Prognostics for Rolling Element Bearings. Mechanical Systems and Signal Processing, 14(5), 747-762.

Liang, S. Y., Li, Y., Billington, S. A., Zhang, C., Shiroishi, J., Kurfess, T. R., \& Danyluk, S. (2014). Adaptive prognostics for rotary machineries. Procedia Engineering, 86, 852-857.

Liao, L. (2014). Discovering prognostic features using genetic programming in remaining useful life prediction. IEEE Transactions on Industrial Electronics, 61(5), 2464-2472.

Liao, L., \& Köttig, F. (2014). Review of hybrid prognostics approaches for remaining useful life prediction of engineered systems, and an application to battery life prediction. IEEE Transactions on Reliability, 63(1), 191-207.

Liao, L., \& Köttig, F. (2016). A hybrid framework combining data-driven and model-based methods for system remaining useful life prediction. Applied Soft Computing Journal, 44, 191-199.

Lim, C. K. R., \& Mba, D. (2015). Switching Kalman filter for failure prognostic. Mechanical Systems and Signal Processing, 52-53(1), 426-435.

Ling, L., Xiao, X., Xiong, J., Zhou, L., Wen, Z., \& Jin, X. (2014). A 3D model for coupling dynamics analysis of high-speed train/track system. Journal of Zhejiang University SCIENCE A, 15(12), 964-983.

Liu, D., Pang, J., Zhou, J., Peng, Y., \& Pecht, M. (2013). Prognostics for state of health estimation of lithiumion batteries based on combination Gaussian process functional regression. Microelectronics Reliability, 53(6), 832-839.

Liu, J., Djurdjanovic, D., Ni, J., Casoetto, N., \& Lee, J. (2007). Similarity based method for manufacturing process performance prediction and diagnosis. Computers in Industry, 58(6), 558-566.

Liu, J., Zhang, M., Zuo, H., \& Xie, J. (2014). Remaining useful life prognostics for aeroengine based on superstatistics and information fusion. Chinese Journal of Aeronautics, 27(5), 1086-1096. 
Liu, J., \& Zio, E. (2016). System dynamic reliability assessment and failure prognostics. Reliability Engineering \& System Safety.

Liu, K., Gebraeel, N. Z., \& Shi, J. (2013). A Data-level fusion model for developing composite health indices for degradation modeling and prognostic analysis. IEEE Transactions on Automation Science and Engineering, 10(3), 652-664.

Liu, L., Wang, S., Liu, D., Zhang, Y., \& Peng, Y. (2015). Entropy-based sensor selection for condition monitoring and prognostics of aircraft engine. Microelectronics Reliability, 55(9-10), 2092-2096.

Long, B., Xian, W., Jiang, L., \& Liu, Z. (2013). An improved autoregressive model by particle swarm optimization for prognostics of lithium-ion batteries. Microelectronics Reliability, 53(6), 821-831.

Marble, S., \& Morton, B. P. (2006). Predicting the Remaining Life of Propulsion System Bearings. 2006 IEEE Aerospace Conference, 1-8.

Marine Jouin n, Rafael Gouriveau, Daniel Hissel, MarieCécile Péra, N. Z. (2016). Particle filter-based prognostics.Review, discussion and perspectives.

Mathew, S., Alam, M., \& Pecht, M. (2012). Identification of Failure Mechanisms to Enhance Prognostic Outcomes. Journal of Failure Analysis and Prevention, 12(1), 66-73.

Mei, T. X., \& Ding, X. J. (2008). New condition monitoring techniques for vehicle suspensions. Railway Condition Monitoring, 2008 4th IET International Conference on, 1-6.

Melnik, R., Sowi, B., Melnik, R., \& Sowi, B. (2014). The Selection Procedure of Diagnostic Indicator of Suspension Fault Modes for the Rail Vehicles Monitoring. EWSHM - 7th European Workshop on Structural Health Monitoring, 159-166.

Mohamed Daowd, Noshin Omar, Bavo Verbrugge, Peter Van Den Bossche, J. V. M. (2010). Battery Models Parameter Estimation based on Matlab: Simulink. The 25th World Battery, Hybrid and Fuel Cell Electric Vehicle Symposium \& Exhibition, 2.

Morgado, T. L. M., Branco, C. M., \& Infante, V. (2008). A failure study of housing of the gearboxes of series 2600 locomotives of the Portuguese Railway Company. Engineering Failure Analysis, 15(1-2), 154-164.

Niu, G., \& Yang, B. S. (2010). Intelligent condition monitoring and prognostics system based on datafusion strategy. Expert Systems with Applications, $37(12), 8831-8840$.

Orsagh, R. F., Sheldon, J., \& Klenke, C. J. (2003). Prognostics/diagnostics for gas turbine engine bearings. IEEE Aerospace Conference Proceedings,

\section{7, 3095-3103}

Paris, P., \& Erdogan, F. (1963). A Critical Analysis of Crack Propagation Laws. Journal of Basic Engineering, 85(4), 528.

Peng, Y., \& Dong, M. (2011). A hybrid approach of HMM and grey model for age-dependent health prediction of engineering assets. Expert Systems with Applications, 38(10), 12946-12953.

Pennacchi, P., Chatterton, S., Vania, A., Ricci, R., \& Borghesani, P. (2013). Experimental evidences in bearing diagnostics for traction system of high speed trains. Chemical Engineering Transactions, 33, 739744.

Pennacchi, P., \& Vania, A. (2008). Diagnostics of a crack in a load coupling of a gas turbine using the machine model and the analysis of the shaft vibrations. Mechanical Systems and Signal Processing, 22(5), $1157-1178$

Pillai, P., Kaushik, A., Bhavikatti, S., Roy, A., \& Kumar, V. (2016). A Hybrid Approach for Fusing Physics and Data for Failure Prediction. International Journal of Prognostics and Health Management, 2153-2648.

Piyush Tagade a, Krishnan S. Hariharan a, Priya Gambhire a, S. M. K. a, \& Taewon Song b, Dukjin Oh b, Taejung Yeo b, S. D. b. (2016). Recursive Bayesian filtering framework for lithium-ion cell state estimation. Journal of Power Sources.

Qiao, G., \& Weiss, B. A. (2016). Advancing Measurement Science to Assess Monitoring, Diagnostics, and Prognostics for Manufacturing Robotics. International Journal of Prognostics and Health Management, 7(Spec Iss on Smart Manufacturing PHM)

Rezvanizaniani, S. M., Liu, Z., Chen, Y., \& Lee, J. (2014) Review and recent advances in battery health monitoring and prognostics technologies for electric vehicle (EV) safety and mobility. Journal of Power Sources, 256, 110-124.

Sai Sarathi Vasan, A., Chen, C., \& Pecht, M. (2013). A Circuit-Centric Approach to Electronic SystemLevel Diagnostics and Prognostics. Prognostics and Health Management (PHM), 2013 IEEE Conference on, 1-8.

Sankararaman, S. (2015). Significance, interpretation, and quantification of uncertainty in prognostics and remaining useful life prediction. Mechanical Systems and Signal Processing, 52-53(1), 228-247.

Sankararaman, S., \& Goebel, K. (2013). A Novel Computational Methodology for Uncertainty Quantification in Prognostics Using The Most Probable Point Concept. Annual Conference of the Prognostics and Health Management Society 2013 
$1-13$.

Sankavaram, C., Kodali, A., Pattipati, K., Singh, S., Zhang, Y., \& Salman, M. (2016). An Inference-based Prognostic Framework for Health Management of Automotive Systems. International Journal of Prognostics and Health Management, 2153-2648.

Satish, B., \& Sarma, N. D. R. (2005). A fuzzy BP approach for diagnosis and prognosis of bearing faults in induction motors. IEEE Power Engineering Society General Meeting 2005, 1-4.

Saxena, A., Celaya, J., Saha, B., Saha, S., \& Goebel, K. (2009). Evaluating algorithm performance metrics tailored for prognostics. IEEE Aerospace Conference Proceedings.

Shahidi, P., Maraini, D., Hopkins, B., \& Seidel, A. (2014). Estimation of Bogie Performance Criteria Through On-Board Condition Monitoring. International Journal of Prognostics and Health Management, 110.

Shahidi, P., Maraini, D., Hopkins, B., \& Seidel, A. (2015). Railcar Bogie Performance Monitoring using Mutual Information and Support Vector Machines. Annual Conference of the Prognostics and Health Management Society, 1-10.

Sharma, V., \& Parey, A. (2016). A Review of Gear Fault Diagnosis Using Various Condition Indicators. Procedia Engineering, 144, 253-263.

Skarlatos, D., Karakasis, K., \& Trochidis, A. (2004). Railway wheel fault diagnosis using a fuzzy-logic method. Applied Acoustics, 65(10), 951-966.

Skima, H., Medjaher, K., Varnier, C., Dedu, E., \& Bourgeois, J. (2016a). A hybrid prognostics approach for MEMS: From real measurements to remaining useful life estimation. Microelectronics Reliability, 65, 79-88.

Skima, H., Medjaher, K., Varnier, C., Dedu, E., \& Bourgeois, J. (2016b). A hybrid prognostics approach for MEMS: From real measurements to remaining useful life estimation. Microelectronics Reliability.

Sun, J., Zuo, H., Wang, W., \& Pecht, M. G. (2014). Prognostics uncertainty reduction by fusing on-line monitoring data based on a state-space-based degradation model. Mechanical Systems and Signal Processing, 45(2), 396-407.

Swanson, D. C. (2001). A general prognostic tracking algorithm for predictive maintenance. 2001 IEEE Aerospace Conference Proceedings (Cat. No.01TH8542), 6, 2971-2977.

Symonds, N., Corni, I., Wood, R. J. K., Wasenczuk, A., \& Vincent, D. (2015). Observing early stage rail axle bearing damage. Engineering Failure Analysis, 56,
$216-232$

Tobon-Mejia, D. A., Medjaher, K., \& Zerhouni, N. (2012). $\mathrm{CNC}$ machine tools wear diagnostic and prognostic by using dynamic Bayesian networks. Mechanical Systems and Signal Processing, 28, 167-182.

Tobon-Mejia, D. A., Medjaher, K., Zerhouni, N., \& Tripot, G. (2012). A data-driven failure prognostics method based on mixture of Gaussian hidden Markov models. IEEE Transactions on Reliability, 61(2), 491-503.

Tsunashima, H., \& Mori, H. (2010). Condition monitoring of railway vehicle suspension using adaptive multiple model approach. Control Automation and Systems (ICCAS), 2010 International Conference on, 584-589.

Virkler, D. A., Hillberry, B. M., \& Goel, P. K. (1979). The Statistical Nature of Fatigue Crack Propagation. Journal of Engineering Materials and Technology, 101(2), 148.

Wang, P., Youn, B. D., \& Hu, C. (2012). A generic probabilistic framework for structural health prognostics and uncertainty management. Mechanical Systems and Signal Processing, 28, 622637.

Wang, Q., Su, Z., \& Hong, M. (2014). Online Damage Monitoring for High-Speed Train Bogie Using Guided Waves: Development and Validation. 7th European Workshop on Structural Health Monitoring July 8-11, 2014. La Cité, Nantes, France.

Wei, C., Xin, Q., Chung, W. H., Liu, S. Y., Tam, H. Y., \& Ho, S. L. (2012). Real-time train wheel condition monitoring by fiber Bragg grating sensors. International Journal of Distributed Sensor Networks, 2012.

Wei Wu, J. H. and J. Z. (2007). Prognostics of Machine Health Condition using an Improved ARIMA-based Prediction method. 2007 2nd IEEE Conference on Industrial Electronics and Applications, 1062-1067.

Williard, N., He, W., Osterman, M., \& Pecht, M. (2013) Comparative analysis of features for determining state of health in lithium-ion batteries. Int. J. Progn. Health Manag, 2013(4), 1-7.

Wu, Y., Jiang, B., Lu, N., \& Zhou, D. (2015). ToMFIRbased Incipient Fault Detection and Estimation for High-speed Rail Vehicle Suspension System. Journal of the Franklin Institute, 352(4), 1672-1692.

Xie, G., Ye, M., Hei, X., Zhao, J., \& Qian, F. (2015). DataBased Health State Analysis for the Axle of High Speed Train. 2015 11th International Conference on Computational Intelligence and Security (CIS), 454 457.

Yang, W. A., Xiao, M., Zhou, W., Guo, Y., \& Liao, W. 
(2016). A hybrid prognostic approach for remaining useful life prediction of lithium-ion batteries. Shock and Vibration, 2016.

Yi, C., Lin, J., Zhang, W., \& Ding, J. (2015). Faults diagnostics of railway axle bearings based on IMF's confidence index algorithm for ensemble EMD. Sensors, 15(5), 10991-11011.

Yu, J. (2011). Bearing performance degradation assessment using locality preserving projections and Gaussian mixture models. Mechanical Systems and Signal Processing, 25(7), 2573-2588.

Zerbst, U., Beretta, S., Kohler, G., Lawton, A., Vormwald, M., Beier, H. T., ... Klingbeil, D. (2013). Safe life and damage tolerance aspects of railway axles - A review. Engineering Fracture Mechanics, 98(1), 214-271.

Zhang, B., Tan, A. C. C., \& Lin, J. hui. (2016). Gearbox fault diagnosis of high-speed railway train. Engineering Failure Analysis, 66, 407-420.

Zhang, G. (2005). Optimum Sensor Localization/Selection In A Diagnostic/Prognostic Architecture. Georgia Institute of Technology, Dissertation, (January).

Zhao, F., Tian, Z., \& Zeng, Y. (2013). Uncertainty quantification in gear remaining useful life prediction through an integrated prognostics method. IEEE Transactions on Reliability, 62(1), 146-159.

Zhu, J., Nostrand, T., Spiegel, C., \& Morton, B. (2014). Survey of Condition Indicators for Condition Monitoring Systems. Annual Conference of the Prognostics and Health Management Society, 5, 113.

Zio, E., \& Peloni, G. (2011). Particle filtering prognostic estimation of the remaining useful life of nonlinear components. Reliability Engineering \& System Safety, 96(3), 403-409. 